\title{
HOMOGENIZATION OF A SEMILINEAR PARABOLIC PDE WITH LOCALLY PERIODIC COEFFICIENTS: A PROBABILISTIC APPROACH
}

\author{
Abdellatif Benchérif-Madani ${ }^{1}$ And Étienne Pardoux ${ }^{2}$
}

\begin{abstract}
In this paper, a singular semi-linear parabolic PDE with locally periodic coefficients is homogenized. We substantially weaken previous assumptions on the coefficients. In particular, we prove new ergodic theorems. We show that in such a weak setting on the coefficients, the proper statement of the homogenization property concerns viscosity solutions, though we need a bounded Lipschitz terminal condition.
\end{abstract}

Mathematics Subject Classification. 35B27, 60H30, 60J60, 60J35.

Received March 28, 2006. Revised December 6, 2006.

\section{IntRoduction}

In this paper we continue investigating locally periodic homogenization in the case of the following semi-linear parabolic PDE defined on the product set $[0, T] \times R^{d}, T>0$,

$$
\left\{\begin{aligned}
\partial_{t} u^{\epsilon}(t, x)+\Gamma^{\epsilon}\left(\frac{x}{\epsilon}, x, u^{\epsilon}(t, x), \nabla u^{\epsilon}(t, x), \partial^{2} u^{\epsilon}(t, x)\right) & =0, \\
u^{\epsilon}(T, x) & =g(x)
\end{aligned}\right.
$$

where

$$
\begin{aligned}
\Gamma^{\epsilon}\left(\frac{x}{\epsilon}, x, u^{\epsilon}(t, x), \nabla u^{\epsilon}(t, x), \partial^{2} u^{\epsilon}(t, x)\right)= & \frac{1}{2} \sum_{i, j=1}^{d} a_{i j}\left(\frac{x}{\epsilon}, x\right) \partial_{x_{i} x_{j}}^{2} u^{\epsilon}(t, x) \\
& +\sum_{i=1}^{d}\left(\frac{1}{\epsilon} b_{i}+b_{i}^{\prime}\right)\left(\frac{x}{\epsilon}, x\right) \partial_{x_{i}} u^{\epsilon}(t, x) \\
& +\frac{1}{\epsilon} e\left(\frac{x}{\epsilon}, x, u^{\epsilon}(t, x)\right)+f\left(\frac{x}{\epsilon}, x, u^{\epsilon}(t, x), \nabla u^{\epsilon}(t, x) \sigma\left(\frac{x}{\epsilon}, x\right)\right)
\end{aligned}
$$

for all $\epsilon>0$ and $x$ in $R^{d}$. The function $g(x)$ is Lipschitz continuous and bounded. All the coefficients are periodic with respect to the first variable with period one in each direction of $R^{d}$. The matrix $\sigma\left(x^{1}, x^{2}\right)=\left[\sigma_{i j}\left(x^{1}, x^{2}\right)\right]$

Keywords and phrases. Homogenization, nonlinear parabolic PDE, Poisson equation, diffusion approximation, backward SDE.

1 Université Ferhat Abbas, Fac. Sciences, Dépt. Maths., Sétif 19000, Algeria; lotfi_madani@yahoo.fr

${ }^{2}$ CMI, LATP - CNRS and Université de Provence, 39, rue F. Joliot Curie, 13453 Marseille Cedex 13, France; pardoux@latp.unimrs.fr (C) EDP Sciences, SMAI 2007 
for $x^{1}$ and $x^{2}$ in $R^{d}$ satisfies $\sigma \sigma^{*}\left(x^{1}, x^{2}\right)=a\left(x^{1}, x^{2}\right)$ where the matrix $a\left(x^{1}, x^{2}\right)=\left[a_{i j}\left(x^{1}, x^{2}\right)\right]$ is supposed to be uniformly elliptic. That is $\exists \lambda$ strictly positive and finite s.t. for all $x^{1}, x^{2}$ and $\xi$ in $R^{d}$

$$
\left(a\left(x^{1}, x^{2}\right) \xi, \xi\right) \geq \lambda\|\xi\|^{2} .
$$

Our previous (probabilistic) arguments in [2] and [3] in the case of linear coefficients $e\left(x / \epsilon, x, u^{\epsilon}(t, x)\right)=$ $e(x / \epsilon, x) u^{\epsilon}(t, x)$ and vanishing non-linear term $f=0$ were based on the Feynman-Kac formula. It is well known that a natural extension of this technique to the non-linear case turns out to be the theory of backward stochastic differential equations first discovered by Pardoux and Peng, see [13], and the references therein, for a complete and profound account. The operator acting on $x$ in $R^{d}$

$$
L^{\epsilon}=\frac{1}{2} \sum_{i, j=1}^{d} a_{i j}\left(\frac{x}{\epsilon}, x\right) \partial_{x_{i} x_{j}}^{2}+\sum_{i=1}^{d}\left(\frac{1}{\epsilon} b_{i}+b_{i}^{\prime}\right)\left(\frac{x}{\epsilon}, x\right) \partial_{x_{i}}
$$

which appears in (1.1) is the infinitesimal generator of the following (forward) SDE, built on some probability space $\left(\Omega, \mathcal{F}_{,} \mathcal{F}_{t}, B_{t}, P\right)$, where $B_{t}$ is under $P$ an $\mathcal{F}_{t}$-Brownian motion,

$$
X_{s}^{\epsilon, t, x}=x+\int_{t}^{s}\left(\frac{1}{\epsilon} b+b^{\prime}\right)\left(\frac{X_{r}^{\epsilon, t, x}}{\epsilon}, X_{r}^{\epsilon, t, x}\right) \mathrm{d} r+\int_{t}^{s} \sigma\left(\frac{X_{r}^{\epsilon, t, x}}{\epsilon}, X_{r}^{\epsilon, t, x}\right) \mathrm{d} B_{r},
$$

for all $0 \leq t \leq s \leq T$ and $x$ in $R^{d}$; whereas the non-linear term is the coefficient in the backward SDE, carried by the above probability space, see the notation below,

$$
Y_{s}^{\epsilon, t, x}=g\left(X_{T}^{\epsilon, t, x}\right)+\int_{s}^{T}\left(\frac{1}{\epsilon} e+f\right)\left(\frac{X_{r}^{\epsilon, t, x}}{\epsilon}, X_{r}^{\epsilon, t, x}, Y_{r}^{\epsilon, t, x}, Z_{r}^{\epsilon, t, x}\right) \mathrm{d} r-\int_{s}^{T} Z_{r}^{\epsilon, t, x} \mathrm{~d} B_{r} ;
$$

the process $\left(Y^{\epsilon, t, x}, Z^{\epsilon, t, x}\right)$ is $\mathcal{F}^{B}$-adapted and subject to the condition

$$
E\left(\sup _{t \leq s \leq T}\left|Y_{s}^{\epsilon, t, x}\right|^{2}\right)+E \int_{t}^{T}\left\|Z_{r}^{\epsilon, t, x}\right\|^{2} \mathrm{~d} r<\infty
$$

for all $\epsilon>0$. Note that $u^{\epsilon}(t, x)=Y_{t}^{\epsilon, t, x}$ is deterministic and solves (1.1) given our assumptions on the coefficients. Our aim in this paper is to establish, under weak conditions on the coefficients, the pointwise convergence as $\epsilon \rightarrow 0$ of $u^{\epsilon}$ towards the solution of equation (3.6) below. For example in [12], who deals with the totally periodic case, the coefficient $e$ satisfies a kind of an algebraic sub-linear growth in $y$, namely $e(x, y)=e_{0}(x, y)+e_{1}(x) y$ where $e_{0}(x, y)$ is bounded besides taking $f=0$. Note also that although Delarue [8] deals with a quasi-linear equation, he considers only periodic coefficients and his conditions concerning $\sigma, b$ and $e$ are much stronger than ours. Moreover, the functions that are homogenized possess weak second derivatives. However, his treatment of the homogenization process is more complete due to the presence of more regularity. On the other hand, although we don't allow for a quadratic growth in the gradient, our work does considerably relax some of the regularity hypotheses of [4], where homogenization is studied by purely analytical methods.

Some facts from [3] will be used here, sometimes without special warnings.

The idea is still to freeze the slow component in (1.3) and consider the following family of operators indexed by $x^{2}$ (since the coefficient $b^{\prime}$ plays no asymptotic role it is omitted),

$$
L_{x^{2}}=\frac{1}{2} \sum_{i, j=1}^{d} a_{i j}\left(x^{1}, x^{2}\right) \partial_{x_{i}^{1} x_{j}^{1}}^{2}+\sum_{i=1}^{d} b_{i}\left(x^{1}, x^{2}\right) \partial_{x_{i}^{1}} .
$$

These operators generate the following diffusions with transition densities $p_{t}\left(x^{1}, x^{1 \prime}, x^{2}\right)$

$$
X_{t}^{x^{2}}=x^{1}+\int_{0}^{t} b\left(X_{r}^{x^{2}}, x^{2}\right) \mathrm{d} r+\int_{0}^{t} \sigma\left(X_{r}^{x^{2}}, x^{2}\right) \mathrm{d} B_{r}
$$


which may rather be thought of as diffusions on the compact torus $T^{d}$, i.e.

$$
\dot{X}_{t}^{x^{2}}=x^{1}+\int_{0}^{t} b\left(\dot{X}_{r}^{x^{2}}, x^{2}\right) \mathrm{d} r+\int_{0}^{t} \sigma\left(\dot{X}_{r}^{x^{2}}, x^{2}\right) \mathrm{d} B_{r}
$$

with transition densities

$$
\dot{p}_{t}\left(x^{1}, x^{1 \prime}, x^{2}\right)=\sum_{k_{1} \ldots k_{d}} p_{t}\left(x^{1}, x^{1 \prime}+\sum_{i=1}^{d} k_{i} e_{i}, x^{2}\right),
$$

$e_{i}$ being the canonical basis of $R^{d}$ and $k_{i}$ integers. In what follows we shall drop the dots when no ambiguity arises. These diffusions possess invariant probability measures $\mu\left(\mathrm{d} x^{1}, x^{2}\right)$ with densities $p_{\infty}\left(x^{1}, x^{2}\right)$, see Pardoux [12]. It is crucial to impose on the singular coefficients $b$ and $e$ the following centering condition for all $x^{2}$ and $y$. As this relation appears several times below and to avoid unnecessary repetitions, we shall use the general notation $h$ for $b, e$ and other functions of interest, namely we have

$$
\int_{\mathbb{T}^{d}} h\left(x^{1}, x^{2}, y\right) \mu\left(\mathrm{d} x^{1}, x^{2}\right)=0
$$

We can then solve the Poisson equation

$$
L_{x^{2}} \widehat{h}\left(x^{1}, x^{2}, y\right)=-h\left(x^{1}, x^{2}, y\right)
$$

and carry on with the usual line of proof (see e.g. the introduction in [2]), provided we have sufficient regularity. Recall that in the linear case we first establish a tightness result for the family of processes $X^{\epsilon}, \epsilon>0$, in the space $\mathcal{C}\left([0, T], R^{d}\right)$ endowed with the sup-norm and proceed to identify the limit via an ergodic theorem and a martingale problem formulation. In the non-linear case however, it seems difficult to work out tightness results for the process $Y^{\epsilon}$ (and the related martingale $M^{\epsilon}$, see (2.17)) in $\mathcal{C}\left([0, T], R^{d}\right)$ endowed with the sup-norm and it turns out that the weaker topology of Jakubowski [9] on $\mathcal{D}\left([0, T], R^{d+1}\right)$ is convenient, see also [11] where a tightness criterion is established (actually relaxed by Kurtz [10]). Moreover, it is important to note that given our formal assumptions on the coefficients, a natural stability argument, first devised in [5] and used below with a slight modification, seems to be necessary since the family of processes $Z^{\epsilon}, \epsilon>0$, does not seem to converge. In particular, Pardoux's weak convergence scheme can't be carried out as such. Instead, we simply begin by guessing the form of the limit PDE (see (3.6)) and then prove that convergence takes place. The whole procedure below for homogenizing our PDE (1.1) should not be too surprising since there seems indeed to be a gap in the bridge between viscosity solutions and BSDEs. This is well accounted for in e.g. [1].

Notation 1.1. Let $\xi$ denote any coefficient $a, b, b^{\prime}, e$ and $f$. Even if the coefficients $a, b$ and $b^{\prime}$ do not depend on $y$ we still allow for the notation $\xi(r, r, r, r)$ meaning respectively $\xi\left(\frac{X_{r}^{\epsilon}}{\epsilon}, X_{r}^{\epsilon}\right), \xi\left(\frac{X_{r}^{\epsilon}}{\epsilon}, X_{r}^{\epsilon}, Y_{r}^{\epsilon}\right)$ and $\xi\left(\frac{X_{r}^{\epsilon}}{\epsilon}, X_{r}^{\epsilon}, Y_{r}^{\epsilon}, Z_{r}^{\epsilon}\right)$. There should be no confusion with space variables since we use different letters for these variables. However, in a solution $u(r, r)$ of a parabolic equation the first $r$ obviously refers to the time variable. We will also drop unambiguous superscripts like e.g. $(t, x)$. The quantity $\zeta(t)-\zeta(s)$ is shortened, using the difference operator, as $\Delta_{s, t} \zeta($.$) . The linear space of R^{d}$ valued continuous, respectively càdlàg, functions on $[0, T]$ is denoted by $\mathcal{C}\left([0, T], R^{d}\right)$, respectively $\mathcal{D}\left([0, T], R^{d}\right)$. If $u(x)$ is a function of $x$ in $R^{d}$, we shall write $\partial_{x} u(x)$ to denote the $d$-dimensional vector whose $i$-th coordinate is $\partial_{x_{i}} u(x)$; similarly $\partial_{x}^{2} u(x)$ will denote a $d \times d$ matrix and so on. Unimportant constants will invariably be designated by $c$ the value of which may vary from line to line while proofs are in process but when there are many constants within a string of relations, we will use $c, c^{\prime} \ldots$ 


\subsection{Condition A}

Our standing assumptions are in this section, next to (1.2) in which $\lambda$ is fixed,

$$
\begin{array}{r}
\int_{\mathbb{T}^{d}} b\left(x^{1}, x^{2}\right) \mu\left(\mathrm{d} x^{1}, x^{2}\right)=0, \\
\int_{\mathbb{T}^{d}} e\left(x^{1}, x^{2}, y\right) \mu\left(\mathrm{d} x^{1}, x^{2}\right)=0,
\end{array}
$$

for all $\left(x^{1}, x^{2}, y\right)$, and $g \in W^{1, \infty}$, the conditions:

\subsubsection{A1 Regularity}

The coefficients $\sigma, b$, and $b^{\prime}$ satisfy a global Lipschitz condition, i.e. there exists a constant $c$ s.t. for any $\xi=\sigma, b$, and $b^{\prime}$

$$
\left\|\xi\left(x^{1}, x^{2}\right)-\xi\left(x^{1 \prime}, x^{2 \prime}\right)\right\| \leq c\left(\left\|x^{1}-x^{1 \prime}\right\|+\left\|x^{2}-x^{2 \prime}\right\|\right) .
$$

Moreover, for $\xi=a$ and $b$, the partial derivatives $\partial_{x^{2}} \xi\left(x^{1},.\right)$ belong to $W_{l o c}^{1, p}\left(R^{d}\right)$, for some large $p$ and all $x^{1} \in \mathbb{T}^{d}$, and

$$
\sup _{x^{2} \in R^{d}}\left\|\partial_{x^{2}}^{2} \xi\left(., x^{2}\right)\right\|_{L^{p}\left(T^{d}\right)}<\infty .
$$

The first order partials of the coefficient $e\left(x^{1}, x^{2}, y\right)$ exist and satisfy for some $c^{\prime}>0$ and all triples $\left(x^{1}, x^{2}, y\right)$

$$
\begin{aligned}
\left(\left\|\partial_{x^{1}} e\right\|+\left\|\partial_{x^{2}} e\right\|\right)\left(x^{1}, x^{2}, y\right) & \leq c^{\prime}(1+|y|), \\
\left|\partial_{y} e\right|\left(x^{1}, x^{2}, y\right) & \leq c^{\prime} .
\end{aligned}
$$

The second partial derivatives of $e$ with respect to $\left(x^{2}, x^{2}\right),\left(x^{2}, y\right)$ and $(y, y)$ exist and satisfy

$$
\begin{aligned}
\left\|\partial_{x^{2}}^{2} e\left(., x^{2}, y\right)\right\|_{L^{p}\left(T^{d}\right)}+\left\|\partial_{x^{2} y}^{2} e\left(., x^{2}, y\right)\right\|_{L^{p}\left(T^{d}\right)} & \leq c^{\prime \prime} \\
\left\|\partial_{y}^{2} e\left(., x^{2}, y\right)\right\|_{L^{p}\left(T^{d}\right)} & \leq c^{\prime \prime} \frac{1}{(1+|y|)},
\end{aligned}
$$

for some large $p$ and $c^{\prime \prime}>0$.

The coefficient $f\left(x^{1}, x^{2}, y, v\right)$ admits first order partials, which satisfy the condition

$$
\begin{aligned}
\left\|\partial_{x^{1}} f\left(x^{1}, x^{2}, y, v\right)\right\|+\left\|\partial_{x^{2}} f\left(x^{1}, x^{2}, y, v\right)\right\| & \leq c^{\prime \prime \prime}(1+|y|+\|v\|), \\
\left|\partial_{y} f\left(x^{1}, x^{2}, y, v\right)\right| & \leq c^{\prime \prime \prime} \\
\left\|\partial_{v} f\left(x^{1}, x^{2}, y, v\right)\right\| & \leq c^{\prime \prime \prime}
\end{aligned}
$$

for some $c^{\prime \prime \prime}>0$ and all $\left(x^{1}, x^{2}, y, v\right)$ uniformly with respect to $x^{1}$ in $\mathbb{T}^{d}$ and $x^{2}$ in $R^{d}$ and moreover it is jointly continuous.

\subsubsection{A2 Growth}

The coefficients $\sigma, b$ and $b^{\prime}$ are bounded, i.e. there exists a constant $K$ s.t. for any $\xi=\sigma, b, b^{\prime}$

$$
\left\|\xi\left(x^{1}, x^{2}\right)\right\| \leq K
$$

and the coefficients $e$ and $f$ satisfy a sub-linear growth in $(y, v)$,

$$
\left|(e+f)\left(x^{1}, x^{2}, y, v\right)\right| \leq K^{\prime}(1+|y|+\|v\|),
$$

for some $K^{\prime}$ and all $x^{1}$ in $\mathbb{T}^{d}, x^{2}$ in $R^{d}, y$ in $R$ and $v$ in $R^{d}$. 
Note that for every $\epsilon>0$ our conditions guarantee the existence and uniqueness of solutions of the above system of forward-backward SDEs subject to condition (1.6) and that a viscosity solution $u^{\epsilon}(t, x)$ to equation (1.1) exists and is unique, see Theorem 12.3 p. 54 in [1].

\subsection{Technical facts}

We begin with the following simple fact, actually of independent interest, which will be needed below.

Lemma 1.2. Let $(\Omega, \mathcal{F})$ be a measurable space carrying two probability measures $P$ and $\widehat{P}$ s.t. $\widehat{P}$ is absolutely continuous with respect to $P$. If a family of continuous processes $\left\{\xi_{s}^{n}\right\}_{n}, t \leq s \leq T$, is $P$-tight, relative to a fixed metric on $\mathcal{C}[t, T]$, then it is also $\widehat{P}$-tight with respect to this same metric. If moreover the density $\zeta=\frac{\mathrm{d} \widehat{P}}{\mathrm{~d} P}$ is in $L^{2}(P)$ then for some absolute constant $c>0$ we have for any non-negative random variable $\theta$

$$
\widehat{E}(\theta) \leq c \sqrt{E\left(\theta^{2}\right)}
$$

Proof. Fix a topology on $\mathcal{C}[0, T]$ and designate the Radon-Nikodym derivative by $\zeta$. As the family of processes $\xi_{s}^{n}$ is $P$-tight, for any $\delta>0$ there exists a compact $K_{\delta}$ in $\mathcal{C}[0, T]$ s.t. $P\left(\xi^{n} \in K_{\delta}^{c}\right)<\delta$ for all $n$. We also have

$$
\widehat{P}\left(\xi^{n} \in K_{\delta}^{c}\right)=\int_{\left\{\xi^{n} \in K_{\delta}^{c}\right\}} \zeta(\omega) P(\mathrm{~d} \omega),
$$

which implies the first result immediately since $\zeta$ is integrable.

The inequality follows from Cauchy-Schwarz.

On the other hand, let $\alpha$ and $\beta$ be two positive numbers. The following inequality will be used several times below,

$$
2 \alpha \beta \leq \rho \alpha^{2}+\rho^{-1} \beta^{2},
$$

where $\rho$ is a small positive number which may depend on the particular product $\alpha \beta$.

\subsection{A growth lemma}

We need to control the growth in $x$ and $y$ of partial derivatives of $\widehat{b}$ and $\widehat{e}$. We have the

Lemma 1.3. There are constants $c_{0}, c, c^{\prime}, c^{\prime \prime}$ and $c^{\prime \prime \prime}$ s.t. for all $\left(x^{1}, x^{2}, y\right)$ in $T^{d} \times R^{d+1}$

$$
\left(\|\widehat{b}\|+\left\|\partial_{x^{1}} \widehat{b}\right\|+\left\|\partial_{x^{2}} \widehat{b}\right\|+\left\|\partial_{x^{2}}^{2} \widehat{b}\right\|+\left\|\partial_{x^{1} x^{2}}^{2} \widehat{b}\right\|\right)\left(x^{1}, x^{2}\right) \leq c_{0},
$$

and on the other hand

$$
\begin{aligned}
\left(\left|\partial_{y} \widehat{e}\right|+\left\|\partial_{x^{1} y}^{2} \widehat{e}\right\|+\left\|\partial_{x^{2} y}^{2} \widehat{e}\right\|+\left\|\partial_{x^{1} x^{2} y}^{3} \widehat{e}\right\|\right)\left(x^{1}, x^{2}, y\right) & \leq c, \\
\left(\left\|\partial_{x^{1} y y}^{3} \widehat{e}\right\|+\left|\partial_{y}^{2} \widehat{e}\right|\right)\left(x^{1}, x^{2}, y\right) & \leq c^{\prime} \frac{1}{(1+|y|)}
\end{aligned}
$$

and

$$
\left(|\widehat{e}|+\left\|\partial_{x^{1}} \widehat{e}\right\|+\left\|\partial_{x^{2}} \widehat{e}\right\|+\left\|\partial_{x^{1}}^{2} \widehat{e}\right\|+\left\|\partial_{x^{1} x^{2}}^{2} \widehat{e}\right\|+\left\|\partial_{x^{2}}^{2} \widehat{e}\right\|+\left\|\partial_{x^{1} x^{2} x^{2}}^{3} \widehat{e}\right\|\right)\left(x^{1}, x^{2}, y\right) \leq c^{\prime \prime}(1+|y|) .
$$

Finally

$$
\left\|\partial_{x^{2}} p_{\infty}\left(x^{1}, x^{2}\right)\right\| \leq c^{\prime \prime \prime}
$$

Moreover, all these partial derivatives are continuous. 
Proof. It suffices to use the same manipulations as in Lemma 1 in [3] and take derivatives in the following Poisson equation

$$
L_{x^{2}} \widehat{e}\left(x^{1}, x^{2}, y\right)=-e\left(x^{1}, x^{2}, y\right)
$$

for $\left(x^{1}, x^{2}, y\right) \in T^{d} \times R^{d+1}$ and to notice that the right hand-side of the corresponding Poisson equation is always majorized by either an absolute constant or by $c(1+|y|)$. Let us e.g. deal with the estimate of $\partial_{x^{1} x^{2} x^{2}}^{3} \widehat{e}$. We have by our notation convention

$$
\partial_{x^{2}} L_{x^{2}} \widehat{e}+L_{x^{2}} \partial_{x^{2}} \widehat{e}=-\partial_{x^{2}} e .
$$

Since equation (1.9) for $e$ implies that $\widehat{e}\left(., x^{2}, y\right)$ is in $W^{2, \infty}\left(T^{d}\right)$ for all $\left(x^{2}, y\right) \in R^{d+1}$ and the coefficient $a$ is Lipschitz, then $\partial_{x^{2}} \widehat{e}\left(., x^{2}, y\right)$ is also in $W^{2, \infty}\left(T^{d}\right)$ for all $\left(x^{2}, y\right) \in R^{d+1}$ with a norm majorized by $c(1+|y|)$. Next we have

$$
\partial_{x^{2}}^{2} L_{x^{2}} \widehat{e}+2 \partial_{x^{2}} L_{x^{2}} \partial_{x^{2}} \widehat{e}+L_{x^{2}} \partial_{x^{2}}^{2} \widehat{e}=-\partial_{x^{2}}^{2} e
$$

Condition A on $a, b$ and $e$ then gives immediately the result.

The other estimates are carried out in a similar way and are left to the reader.

The estimate on the derivatives with respect to $x^{2}$ of the invariant probability density $p_{\infty}\left(x^{1}, x^{2}\right)$ follows from Lemma 18 in [2].

\section{Removing the SINGUlaRities}

We want to get rid of the factor $\epsilon^{-1}$ in both the forward and backward SDEs above but keeping as few conditions on the coefficients as possible. Our method of attack, in fact Freidlin's (see the introduction in [2]), consists in applying the joint Itô formula on the functions $\widehat{b}$ and $\widehat{e}$, when possible. In that respect, it follows from Lemma 1.3 that $\widehat{b}$ is in $W_{\text {loc }}^{2, \infty}\left(T^{d} \times R^{d}\right)$ and that $\widehat{e}$ is in $W^{2, p}\left(T^{d} \times R^{d+1}\right)$ for some large $p$ which allows the use of the Itô-Krylov formula.

\subsection{Treatment of the forward process}

Let us first define

$$
\begin{aligned}
F^{\prime}\left(x^{1}, x^{2}\right) & =\left(\partial_{x^{1}} \widehat{b} b^{\prime}+\partial_{x^{2}} \widehat{b} b+\operatorname{Tr} \partial_{x^{1} x^{2}}^{2} \widehat{b} a\right)\left(x^{1}, x^{2}\right), \\
G(x, y) & =\left[\left(I+\partial_{x^{1}} \widehat{b}\right) \sigma\right]\left(x^{1}, x^{2}\right),
\end{aligned}
$$

where, see the notation above, the quantity $\operatorname{Tr} \partial_{x^{1} x^{2}}^{2} \widehat{b} a$ stands for the vector whose components are $\operatorname{Tr} \partial_{x^{1} x^{2}}^{2} \widehat{b}_{i} a$, $1 \leq i \leq d$. Recall that $0 \leq t \leq s \leq T$. We have, see [3], the decomposition

$$
X_{s}^{\epsilon}=\bar{X}_{s}^{\epsilon}+R_{s}^{\epsilon}
$$

where

and

$$
\bar{X}_{s}^{\epsilon}=x+\int_{t}^{s} F^{\prime}(r, r) \mathrm{d} r+\int_{t}^{s} G(r, r) \mathrm{d} B_{r}
$$

$$
R_{s}^{\epsilon}=\epsilon\left[\int_{t}^{s}\left(\partial_{x^{2}} \widehat{b} b^{\prime}+\frac{1}{2} T r \partial_{x^{2}}^{2} \widehat{b} a\right)(r, r) \mathrm{d} r+\int_{t}^{s} \partial_{x^{2}} \widehat{b} \sigma(r, r) \mathrm{d} B_{r}+(\widehat{b}(t, t)-\widehat{b}(s, s))\right] .
$$

Note that since $b, b^{\prime}$ and $a$ are bounded we have

$$
E\left(\sup _{s \leq T}\left\|R_{s}^{\epsilon}\right\|^{4}\right) \leq c \epsilon^{4},
$$

whence the tightness (in the sup-norm) sufficient condition (which was proved in [3] under weaker conditions). 
Corollary 2.1. There exists a constant $c$ s.t. for all $0 \leq \alpha \leq \beta \leq T$ and $\epsilon>0$

$$
E\left(\left\|X_{\beta}^{\epsilon}-X_{\alpha}^{\epsilon}\right\|^{4}\right) \leq c\left[(\beta-\alpha)^{2}+\epsilon^{4}\right]
$$

Note that we also have the following estimate which will be needed below

$$
E\left(\left\|X_{\beta}^{\epsilon}-X_{\alpha}^{\epsilon}\right\|^{2}\right) \leq c\left[(\beta-\alpha)+\epsilon^{2}\right]
$$

\subsection{Treatment of the backward process}

In order to remove the singularity of the backward process, we shall use a kind of stopping argument. Let us take a fine enough equidistant subdivision of the interval $[0, T]$ by means of the points $t_{i}, i=0, \ldots,[T / \Delta t]=N$, where $t_{0}=0$ and $\Delta t=t_{i}-t_{i-1}$. We denote by $t_{*}$ the largest $t_{i}$ below $t$, by $t^{*}$ the least $t_{i}$ above $t$. On the last interval $\left[T_{*}, T\right]$ however, we make the convention that for $r \in\left(T_{*}, T\right]$ we have $r^{*}=T\left(\operatorname{instead}\right.$ of $\left.T_{*}+\Delta t\right)$. We also note that $t^{*}=t$ when $t=t_{i}$. We define in particular the subdivisions $\Delta t^{k}=k \epsilon^{2}$, where $k$ is a positive integer, and call $\Delta t^{1}$ the neutral subdivision. We add a superscript (subscript) $k$ to indicate which subdivision is involved. Recall that we have for $s \leq T$

$$
Y_{s}^{\epsilon}=g\left(X_{T}^{\epsilon}\right)+\int_{s}^{T}\left(\frac{1}{\epsilon} e+f\right)\left(r, r, Y_{r}^{\epsilon}, Z_{r}^{\epsilon}\right) \mathrm{d} r-\int_{s}^{T} Z_{r}^{\epsilon} \mathrm{d} B_{r}
$$

Define for $s \leq T$ the discontinuous càdlàg adapted process

$$
\widehat{Y}_{s}^{k, \epsilon}=Y_{s}^{\epsilon}-\epsilon \widehat{e}\left(\frac{X_{s}^{\epsilon}}{\epsilon}, X_{s}^{\epsilon}, Y_{s_{*(k)}}^{\epsilon}\right)
$$

that is the process $\widehat{Y}_{s}^{k, \epsilon}$ is continuous on each $\left(t_{i-1}^{k}, t_{i}^{k}\right)$ and undergoes a jump at each $t_{i}^{k}, t_{1}^{k} \leq t_{i}^{k} \leq T_{*(k)}$ (there should be no ambiguity with these inequalities). We have thanks to the Itô formula,

$$
\begin{aligned}
\int_{s}^{T} \frac{1}{\epsilon} e\left(r, r, Y_{r_{*(k)}}^{\epsilon}\right) \mathrm{d} r= & \epsilon\left[\widehat{e}\left(s, s, Y_{s_{*(k)}}^{\epsilon}\right)-\widehat{e}\left(T, T, Y_{T_{*(k)}}^{\epsilon}\right)\right]+\int_{s}^{T}\left[\partial_{x^{1}} \widehat{e}\left(r, r, r_{*(k)}\right) b^{\prime}(r, r)+\partial_{x^{2}} \widehat{e}\left(r, r, r_{*(k)}\right) b(r, r)\right. \\
& +\operatorname{Tr}_{x^{1} x^{2}}^{2} \widehat{e}\left(r, r, r_{*(k)}\right) a(r, r) \\
& \left.+\epsilon \partial_{x^{2}} \widehat{e}\left(r, r, r_{*(k)}\right) b^{\prime}(r, r)+\frac{\epsilon}{2} \operatorname{Tr}_{x^{2}}^{2} \widehat{e}\left(r, r, r_{*(k)}\right) a(r, r)\right] \mathrm{d} r \\
& +\int_{s}^{T}\left(\partial_{x^{1}} \widehat{e}+\epsilon \partial_{x^{2}} \widehat{e}\right)\left(r, r, r_{*(k)}\right) \sigma(r, r) \mathrm{d} B_{r} \\
& +\epsilon \sum_{t_{i}^{k}>s}^{t_{i}^{k}=T_{*(k)}} \Delta_{t_{i-1}^{k}, t_{i}^{k}} \widehat{e}\left(t_{i}^{k}, t_{i}^{k}, Y_{.}^{\epsilon}\right) .
\end{aligned}
$$

Hence we have the representation

$$
\widehat{Y}_{s}^{k, \epsilon}=\left[g\left(X_{T}^{\epsilon}\right)-\epsilon \widehat{e}\left(T, T, Y_{T_{*(k)}}^{\epsilon}\right]+\int_{s}^{T} \widehat{U}_{r}^{k, \epsilon} \mathrm{d} r-\int_{s}^{T} \widehat{Z}_{r}^{k, \epsilon} \mathrm{d} B_{r}+J_{s}^{k, \epsilon},\right.
$$


where

$$
\begin{aligned}
\widehat{U}_{1, r}^{k, \epsilon}= & \frac{1}{\epsilon} \Delta_{r_{*(k)}, r} e\left(r, r, Y_{.}^{\epsilon}\right), \\
\widehat{U}_{2, r}^{k, \epsilon}= & {\left[\partial_{x^{1}} \widehat{e}\left(r, r, r_{*(k)}\right) b^{\prime}(r, r)+\partial_{x^{2}} \widehat{e}\left(r, r, r_{*(k)}\right) b(r, r)\right.} \\
& \left.+\operatorname{Tr} \partial_{x^{1} x^{2}}^{2} \widehat{e}\left(r, r, r_{*(k)}\right) a(r, r)\right], \\
\widehat{U}_{3, r}^{k, \epsilon}= & {\left[\epsilon \partial_{x^{2}} \widehat{e}\left(r, r, r_{*(k)}\right) b^{\prime}(r, r)+\frac{\epsilon}{2} \operatorname{Tr}_{x^{2}}^{2} \widehat{e}\left(r, r, r_{*(k)}\right) a(r, r)\right], } \\
\widehat{U}_{r}^{k, \epsilon}= & \sum_{i=1}^{3} \widehat{U}_{i, r}^{k, \epsilon}+f\left(r, r, Y_{r}^{\epsilon}, Z_{r}^{\epsilon}\right)
\end{aligned}
$$

and

and finally

$$
\widehat{Z}_{r}^{k, \epsilon}=Z_{r}^{\epsilon}-\left(\partial_{x^{1}} \widehat{e}+\epsilon \partial_{x^{2}} \widehat{e}\right)\left(r, r, r_{*(k)}\right) \sigma(r, r)
$$

$$
J_{s}^{k, \epsilon}=\epsilon \sum_{t_{i}^{k}>s}^{T_{*(k)}} \Delta_{t_{i-1}^{k}, t_{i}^{k}} \widehat{e}\left(t_{i}^{k}, t_{i}^{k}, Y_{.}^{\epsilon}\right) .
$$

\subsection{Establishing tightness}

Having thus produced a non singular process $\widehat{Y}_{s}^{k, \epsilon}$, which depends on $k$ and is asymptotically close to $Y_{s}^{\epsilon}$, let us turn to the study of the tightness problem. Consider the formula (2.4). It is important to notice that in the first time interval $\left[s, s^{*(k)}\right)$ in the Lebesgue (respectively the stochastic) integral the process $\widehat{U}_{r}^{k, \epsilon}$ (respectively the process $\widehat{Z}_{r}^{k, \epsilon}$ ) has the component $Y_{s_{*(k)}}^{\epsilon}$ which stays lagging below $s$. The same phenomenon arises in the first jump term in $J_{s}^{k, \epsilon}$. Therefore estimating the second moment of the process $\widehat{Y}_{s}^{k, \epsilon}$ and the first moment of the related process $\int_{s}^{T}\left\|\widehat{Z}_{r}^{k, \epsilon}\right\|^{2} \mathrm{~d} r$ by the usual methods needs a special care. This will allow us to handle supremums that run backwards in time. Of all the terms in (2.5), it is the first one which requires attention since it has exactely the reverse behaviour of the jump term $J_{s}^{k, \epsilon}$ in (2.4).

\subsubsection{Estimates on the increments of $Y^{\epsilon}$}

Let us begin with the following easy lemma.

Lemma 2.2. Under the above notations, there exists a constant $c>0$ and an $\epsilon_{0}>0$ s.t. for any $k \geq 1, \epsilon \leq \epsilon_{0}$ and $r$ in $[0, T]$, we have

and

$$
\left\|Z_{r}^{\epsilon}\right\| \leq c\left(1+\left|\Delta_{r_{*(k)}, r} Y^{\epsilon}\right|+\left|Y_{r}^{\epsilon}\right|+\left\|\widehat{Z}_{r}^{k, \epsilon}\right\|\right)
$$

$$
\left|\widehat{U}_{r}^{k, \epsilon}\right| \leq c\left(1+\frac{1}{\epsilon}\left|\Delta_{r_{*(k)}, r} Y_{.}^{\epsilon}\right|+\left|Y_{r}^{\epsilon}\right|+\left\|\widehat{Z}_{r}^{k, \epsilon}\right\|\right) .
$$

Proof. The first inequality follows immediately from Lemma 1.3, when $\epsilon \leq 1$ we have

$$
\begin{aligned}
\left\|Z_{r}^{\epsilon}\right\| & \leq c\left(1+\left|Y_{r_{*(k)}}^{\epsilon}\right|+\left\|\widehat{Z}_{r}^{k, \epsilon}\right\|\right) \\
& \leq c\left(1+\left|\Delta_{r_{*(k)}, r} Y^{\epsilon}\right|+\left|Y_{r}^{\epsilon}\right|+\left\|\widehat{Z}_{r}^{k, \epsilon}\right\|\right) .
\end{aligned}
$$

This inequality will serve to recover the process $\left\|\widehat{Z}_{r}^{k, \epsilon}\right\|^{2}$ when we deal with Lebesgue integrals involving the process $\left\|Z_{r}^{\epsilon}\right\|^{2}$.

The other inequality is proved in a similar way and follows from the first inequality (2.7). 
This allows us to control powers of the increments of the process $Y_{s}^{\epsilon}$. We have the

Lemma 2.3. There exists an absolute constant $c>0$ that depends only on the coefficients in Condition $A$ and an $\epsilon_{0}>0$ s.t., when $\epsilon \leq \epsilon_{0}$ we have on each $\Delta t_{i}^{k}, i=1, \ldots, N^{k}+1, k \geq 1$,

$$
\begin{aligned}
\left|Y_{r}^{\epsilon}-Y_{t_{i-1}^{k}}^{\epsilon}\right| \leq & c\left(\Delta t^{k}+\frac{1}{\epsilon} \int_{t_{i-1}^{k}}^{r}\left|Y_{u}^{\epsilon}-Y_{t_{i-1}^{k}}^{\epsilon}\right| \mathrm{d} u+\left\|\Delta_{t_{i-1}^{k}, r} X^{\epsilon}\right\|+\left|Y_{t_{i-1}^{k}}^{\epsilon}\right|\left\|\Delta_{t_{i-1}^{k}, r} X_{.}^{\epsilon}\right\|\right. \\
& \left.+\int_{t_{i-1}^{k}}^{r}\left|Y_{u}^{\epsilon}\right| \mathrm{d} u+\int_{t_{i-1}^{k}}^{r}\left\|\widehat{Z}_{u}^{k, \epsilon}\right\| \mathrm{d} u+\left|\int_{t_{i-1}^{k}}^{r} \widehat{Z}_{u}^{k, \epsilon} \mathrm{d} B_{u}\right|\right) .
\end{aligned}
$$

Proof. Notice first that $e$ is Lipschitz with respect to $y$, that $b$ and $b^{\prime}$ are bounded and that, thanks to Lemma 1.3, there is a constant $c>0$ s.t. for all $\epsilon \leq 1$

$$
\epsilon\left|\Delta_{t_{i-1}^{k}, r} \widehat{e}\left(., ., t_{i-1}^{k}\right)\right| \leq c\left(1+\left|Y_{t_{i-1}^{\epsilon}}^{\epsilon}\right|\right)\left\|\Delta_{t_{i-1}^{k}, r} X_{.}^{\epsilon}\right\|,
$$

then it suffices to use Lemma 2.2 in the Itô-Krylov decomposition (2.4).

Applying the Gronwall-Bellman inequality, we deduce the

Corollary 2.4. Under the same hypotheses as in the previous lemma we have

$$
\begin{aligned}
\left|Y_{r}^{\epsilon}-Y_{t_{i-1}^{k}}^{\epsilon}\right| \leq & c e^{c k \epsilon}\left(\Delta t^{k}+\left\|\Delta_{t_{i-1}^{k}, r} X_{\cdot}^{\epsilon}\right\|+\left|Y_{t_{i-1}^{\epsilon}}^{\epsilon}\right|\left\|\Delta_{t_{i-1}^{k}, r} X_{\cdot}^{\epsilon}\right\|\right. \\
& \left.+\int_{t_{i-1}^{k}}^{r}\left|Y_{u}^{\epsilon}\right| \mathrm{d} u+\int_{t_{i-1}^{k}}^{r}\left\|\widehat{Z}_{u}^{k, \epsilon}\right\| \mathrm{d} u+\left|\int_{t_{i-1}^{k}}^{r} \widehat{Z}_{u}^{k, \epsilon} \mathrm{d} B_{u}\right|\right)
\end{aligned}
$$

and the

Corollary 2.5. There exists an absolute constant $c>0$ which depends only on the coefficients in Condition $A$ s.t., for any fixed $k \geq 1$ there is an $\epsilon_{0}(k)>0$ s.t. when $\epsilon \leq \epsilon_{0}$ we have on each $\Delta t_{i}^{k}, i=1, \ldots, N^{k}+1$,

$$
E \sup _{r \in \Delta t_{i}^{k}}\left|Y_{r}^{\epsilon}-Y_{t_{i-1}^{k}}^{\epsilon}\right|^{2} \leq c\left(\Delta t^{k}+\int_{\Delta t_{i}^{k}} E\left|Y_{u}^{\epsilon}\right|^{2} \mathrm{~d} u+\Delta t^{k} E\left|Y_{t_{i-1}^{k}}^{\epsilon}\right|^{2}+E \int_{\Delta t_{i}^{k}}\left\|\widehat{Z}_{u}^{k, \epsilon}\right\|^{2} \mathrm{~d} u\right) .
$$

Proof. Since the coefficients of the forward equation are bounded, it suffices to write thanks to the relation (2.2) and to Lemma 1.3

$$
\left|Y_{t_{i-1}^{k}}^{\epsilon}\right|\left\|\Delta_{t_{i-1}^{k}, r} X_{.}^{\epsilon}\right\| \leq c\left(\epsilon+\Delta t^{k}\right)\left|Y_{t_{i-1}^{k}}^{\epsilon}\right|+\left\|\int_{t_{i-1}^{k}}^{r}\left|Y_{t_{i-1}^{k}}^{\epsilon}\right| \widehat{G}(u, u) \mathrm{d} B_{u}\right\|,
$$

where

$$
\widehat{G}(u, u)=\left(I+\partial_{x^{1}} \widehat{b}+\epsilon \partial_{x^{2}} \widehat{b}\right)(u, u) .
$$


By the Burkholder-Davis-Gundy inequality, we see that for any given $k$, we can choose an $\epsilon_{0}$ s.t. for all $\epsilon \leq \epsilon_{0}$ we have

$$
E \sup _{r \in \Delta t_{i}^{k}}\left\|\left|Y_{t_{i-1}^{k}}^{\epsilon}\right| \Delta_{t_{i-1}^{k}, r} X_{.}^{\epsilon}\right\|^{2} \leq c\left(1+\Delta t^{k}\right) \Delta t^{k} E\left|Y_{t_{i-1}^{k}}^{\epsilon}\right|^{2} \leq c^{\prime} \Delta t^{k} E\left|Y_{t_{i-1}^{k}}^{\epsilon}\right|^{2}
$$

Using convexity, the inequalities of Cauchy-Schwarz and Burkholder-Davis-Gundy on the relation of the above corollary we arrive at the desired result, upon taking $\epsilon$ small enough.

Remark 2.6. Notice that in both the previous corollaries, the time $r$ can take the extreme value $t_{i}^{k}$.

Before we work out the usual a priori estimates, let us first settle the problems that we mentionned at the beginning if this subsection. We formulate our treatment as an independent

Lemma 2.7. There exists a constant $c>0$ s.t., for any fixed $k>0$ there is an $\epsilon_{0}(k)>0$ s.t. for all $\epsilon \leq \epsilon_{0}$ and $s \leq T$

$$
E \sup _{s_{*(k)} \leq r \leq s^{*(k)}}\left|\Delta_{s_{*(k)}, r} Y_{.}^{\epsilon}\right|^{2} \leq c\left(1+\sup _{s \leq r \leq T} E\left|\widehat{Y}_{r}^{k, \epsilon}\right|^{2}\right)
$$

Proof. We can write by Corollary 2.5

$$
\begin{aligned}
E \sup _{s_{*(k)} \leq r \leq s^{*(k)}}\left|\Delta_{s_{*(k)}, r} Y_{.}^{\epsilon}\right|^{2} & \leq c \Delta t^{k}\left(1+\sup _{s_{*(k)} \leq r \leq s^{*(k)}} E\left|Y_{r}^{\epsilon}\right|^{2}+E \int_{s_{*(k)}}^{s^{*(k)}}\left\|\widehat{Z}_{u}^{k, \epsilon}\right\|^{2} \mathrm{~d} u\right) \\
& \leq c \Delta t^{k}\left(1+\sup _{s_{*(k)} \leq r \leq s^{*(k)}} E\left|Y_{r}^{\epsilon}\right|^{2}+E \int_{s_{*(k)}}^{s^{*(k)}}\left\|Z_{u}^{\epsilon}\right\|^{2} \mathrm{~d} u\right)
\end{aligned}
$$

since by definition of $\widehat{Z}_{u}^{k, \epsilon}$ it follows by Lemma 1.3 that for $\epsilon \leq 1$ and $u$ in $\left[s_{*(k)}, s^{*(k)}\right]$ we have

$$
\left\|\widehat{Z}_{u}^{k, \epsilon}\right\|^{2} \leq c\left(1+\left|Y_{S_{*(k)}}^{\epsilon}\right|^{2}+\left\|Z_{u}^{\epsilon}\right\|^{2}\right)
$$

On the other hand, by Condition A and by a well known BSDE estimate, derived when starting from our crude and untreated BSDE on $\left[s_{*(k)}, s^{*(k)}\right]$, i.e.

$$
Y_{r}^{\epsilon}=Y_{s^{*}(k)}^{\epsilon}+\int_{r}^{s^{*(k)}}\left(\frac{1}{\epsilon} e+f\right)\left(u, u, Y_{u}^{\epsilon}, Z_{u}^{\epsilon}\right) \mathrm{d} u-\int_{r}^{s^{*(k)}} Z_{u}^{\epsilon} \mathrm{d} B_{u},
$$

the right hand-side of the above inequality is majorized, when $\epsilon \leq \epsilon_{0}(k)$, by

$$
c\left(1+E\left|Y_{s^{*}(k)}^{\epsilon}\right|^{2}\right),
$$

since the factor $\frac{1}{\epsilon}$ (more than) cancels out with $\Delta t^{k}=k \epsilon^{2}$.

Next we need to recover the process $\widehat{Y}_{s}^{k, \epsilon}$, that is we have to compare our former process $Y_{s}^{\epsilon}$ with the new one $\widehat{Y}_{s}^{k, \epsilon}$. Recall that we have for all $s \leq T$

$$
Y_{s}^{\epsilon}=\widehat{Y}_{s}^{k, \epsilon}+\epsilon \widehat{e}\left(s, s, Y_{s_{*(k)}}^{\epsilon}\right),
$$


so that by well known inequalities when $\epsilon \leq 1$

$$
\begin{aligned}
E\left|Y_{s}^{\epsilon}\right|^{2} & \leq c\left[E\left|\widehat{Y}_{s}^{k, \epsilon}\right|^{2}+\epsilon^{2}\left(1+E\left|Y_{s_{*(k)}}^{\epsilon}\right|^{2}\right)\right] \\
& \leq c\left(1+E\left|\widehat{Y}_{s}^{k, \epsilon}\right|^{2}+\epsilon^{2} E\left|\Delta_{s_{*(k)}, s^{*(k)}} Y_{\cdot}^{\epsilon}\right|^{2}+\epsilon^{2} E\left|Y_{s^{*}(k)}^{\epsilon}\right|^{2}\right) .
\end{aligned}
$$

We also know from the above manipulation that when $\epsilon$ is small enough

$$
E\left|\Delta_{s_{*(k)}, s^{*(k)}} Y^{\epsilon}\right|^{2} \leq c\left(1+E\left|Y_{s^{*(k)}}^{\epsilon}\right|^{2}\right),
$$

so that

$$
E\left|Y_{s}^{\epsilon}\right|^{2} \leq c\left(1+E\left|\widehat{Y}_{s}^{k, \epsilon}\right|^{2}+\epsilon^{2} E\left|Y_{s^{*}(k)}^{\epsilon}\right|^{2}\right)
$$

Now

which gives for any $s \leq T$

$$
E\left|Y_{s^{*}(k)}^{\epsilon}\right|^{2} \leq \sup _{s \leq r \leq T} E\left|Y_{r}^{\epsilon}\right|^{2}
$$

$$
\left(1-c \epsilon^{2}\right) \sup _{s \leq r \leq T} E\left|Y_{r}^{\epsilon}\right|^{2} \leq c\left(1+\sup _{s \leq r \leq T} E\left|\widehat{Y}_{r}^{k, \epsilon}\right|^{2}\right),
$$

when $\epsilon \leq \epsilon_{0}(k)$, which immediately implies our lemma.

Remark 2.8. The sharp estimate on $E \sup _{s_{*(k)} \leq r \leq s^{*(k)}}\left|\Delta_{s_{*(k)}, r} Y^{\epsilon}\right|^{2}$ given by Corollary 2.5 is not really needed in order to prove the above lemma. A rough and well known estimate based on the untreated BSDE above is sufficient since we can control $k$ thanks to the parameter $\rho_{1}$ as announced in equation (1.10), see below.

2.3.2. A priori estimates

We seek to establish the following relation, see e.g. [11],

$$
\sup _{\epsilon \leq \epsilon_{0}}\left(E \sup _{0 \leq s \leq T}\left|Y_{s}^{\epsilon}\right|^{2}+E \int_{0}^{T}\left\|Z_{r}^{\epsilon}\right\|^{2} \mathrm{~d} r\right)<c,
$$

for some fixed $c>0$ and $\epsilon_{0}$. This fundamental estimate will serve to imply that the family of processes $\left(Y_{s}^{\epsilon}, M_{s}^{\epsilon}\right)$ (see eq. (2.17) below for the definition of the martingale $M_{s}^{\epsilon}$ ) is tight in the Jakubowski $S$-topology. We will use parameters $\rho>0$ which will be used as in equation (1.10).

By the Itô formula, we have for $t \leq s \leq T$

$$
\begin{aligned}
\left|\widehat{Y}_{s}^{k, \epsilon}\right|^{2}+\int_{s}^{T}\left\|\widehat{Z}_{r}^{k, \epsilon}\right\|^{2} \mathrm{~d} r+\sum_{t_{i}^{k}>s}^{T_{*(k)}}\left(\Delta \widehat{Y}_{t_{i}^{k}}^{k, \epsilon}\right)^{2}= & {\left[g\left(X_{T}^{\epsilon}\right)-\epsilon \widehat{e}\left(T, T, Y_{T_{*(k)}}^{\epsilon}\right)\right]^{2}+\int_{s}^{T} 2 \widehat{Y}_{r}^{k, \epsilon} \widehat{U}_{r}^{k, \epsilon} \mathrm{d} r } \\
& -\int_{s}^{T} 2 \widehat{Y}_{r}^{k, \epsilon} \widehat{Z}_{r}^{k, \epsilon} \mathrm{d} B_{r}-\sum_{t_{i}^{k}>s}^{T_{*(k)}} 2 \widehat{Y}_{t_{i}^{k}-}^{k, \epsilon} \cdot \Delta \widehat{Y}_{t_{i}^{k}}^{k, \epsilon},
\end{aligned}
$$

where $\Delta \widehat{Y}_{t_{i}^{k}}^{k, \epsilon}$ stands for the jump of the process $\widehat{Y}_{s}^{k, \epsilon}$ at the instant $t_{i}^{k}$, i.e.

$$
\begin{aligned}
\Delta \widehat{Y}_{t_{i}^{k}}^{k, \epsilon} & =\widehat{Y}_{t_{i}^{k}+}^{k, \epsilon}-\widehat{Y}_{t_{i}^{k}-}^{k, \epsilon} \\
& =\epsilon\left[\widehat{e}\left(t_{i}^{k}, t_{i}^{k}, Y_{t_{i-1}^{k}}^{\epsilon}\right)-\widehat{e}\left(t_{i}^{k}, t_{i}^{k}, Y_{t_{i}^{k}}^{\epsilon}\right)\right]
\end{aligned}
$$


Keep in mind our convention about the stars $*$ in the introduction of this section and apply the expected value operator $E$ on both sides of the above Itô formula. Take $\epsilon \leq 1$, we have thanks to standard inequalities, to Lemmas 1.3 and 2.2 (it is easy to see that the expected value of the stochastic integral vanishes)

$$
\begin{aligned}
& E\left|\widehat{Y}_{s}^{k, \epsilon}\right|^{2}+E \int_{s}^{T}\left\|\widehat{Z}_{r}^{k, \epsilon}\right\|^{2} \mathrm{~d} r+\sum_{t_{i}^{k}>s}^{T_{*(k)}} E\left|\Delta \widehat{Y}_{t_{i}^{k}}^{k, \epsilon}\right|^{2} \leq c\left[1+E\left|g\left(X_{T}^{\epsilon}\right)\right|^{2}+\epsilon^{2} E\left|\Delta_{T_{*(k)}, T} Y_{.}^{\epsilon}\right|^{2}\right. \\
& +\frac{1}{\rho_{1}^{2}} \int_{s}^{s^{*(k)}} E\left|\widehat{Y}_{r}^{k, \epsilon}\right|^{2} \mathrm{~d} r+\rho_{1}^{2} \int_{s}^{s^{*(k)}} \frac{1}{\epsilon^{2}} E\left|Y_{r}^{\epsilon}-Y_{s_{*(k)}}^{\epsilon}\right|^{2} \mathrm{~d} r \\
& +\rho_{1}^{2} \int_{s}^{s^{*(k)}} E\left|Y_{r}^{\epsilon}\right|^{2} \mathrm{~d} r+\rho_{1}^{2} \int_{s}^{s^{*(k)}} E\left\|\widehat{Z}_{r}^{k, \epsilon}\right\|^{2} \mathrm{~d} r \\
& +\frac{1}{\rho_{2}^{2}} \int_{s^{*}(k)}^{T} E\left|\widehat{Y}_{r}^{k, \epsilon}\right|^{2} \mathrm{~d} r+\rho_{2}^{2} \int_{s^{*}(k)}^{T} \frac{1}{\epsilon^{2}} E\left|Y_{r}^{\epsilon}-Y_{r_{*(k)}}^{\epsilon}\right|^{2} \mathrm{~d} r \\
& +\rho_{2}^{2} \int_{s^{*(k)}}^{T} E\left|Y_{r}^{\epsilon}\right|^{2} \mathrm{~d} r+\rho_{2}^{2} \int_{s^{*(k)}}^{T} E\left\|\widehat{Z}_{r}^{k, \epsilon}\right\|^{2} \mathrm{~d} r \\
& \left.+E\left|\widehat{Y}_{s^{*(k)}-}^{k, \epsilon}\right|\left|\Delta \widehat{Y}_{s^{*}(k)}^{k, \epsilon}\right|+\sum_{t_{i}^{k}>s^{*(k)}}^{T_{*(k)}} E\left|\widehat{Y}_{t_{i}^{k}-}^{k, \epsilon}\right|\left|\Delta \widehat{Y}_{t_{i}^{k}}^{k, \epsilon}\right|\right] .
\end{aligned}
$$

Due to the factor $\epsilon^{2}$, the third term on the right above $\epsilon^{2} E\left|\Delta_{T_{*(k)}, T} Y^{\epsilon}\right|^{2}$ is easily treated by Lemma 2.7 , we indeed have

$$
\epsilon^{2} E\left|\Delta_{T_{*(k)}, T} Y_{.}^{\epsilon}\right|^{2} \leq \epsilon^{2} c\left(1+\sup _{s \leq r \leq T} E\left|\widehat{Y}_{r}^{k, \epsilon}\right|^{2}\right)
$$

The fifth and seventh terms on the right hand-side above are also dealt with easily using Lemma 2.7. Next, let us write thanks to Corollary 2.5 and to the inequality (2.8)

$$
\begin{aligned}
\int_{s^{*(k)}}^{T} \frac{1}{\epsilon^{2}} E\left|Y_{r}^{\epsilon}-Y_{r_{*(k)}}^{\epsilon}\right|^{2} \mathrm{~d} r & \leq k \sum_{t_{i}^{k}>s^{*(k)}}^{T^{*(k)}} E \sup _{r \in \Delta t_{i}^{k}}\left|\Delta_{t_{i-1}^{k}, r} Y_{.}^{\epsilon}\right|^{2} \\
& \leq c k\left(1+\int_{s^{*(k)}}^{T} E\left|Y_{r}^{\epsilon}\right|^{2} \mathrm{~d} r+\int_{s^{*(k)}}^{T} E\left\|\widehat{Z}_{r}^{k, \epsilon}\right\|^{2} \mathrm{~d} r+\sup _{s^{*(k)} \leq r \leq T} E\left|Y_{r}^{\epsilon}\right|^{2}\right) \\
& \leq c(k)\left(1+\sup _{s \leq r \leq T} E\left|\widehat{Y}_{r}^{k, \epsilon}\right|^{2}+\int_{s}^{T} E\left\|\widehat{Z}_{r}^{k, \epsilon}\right\|^{2} \mathrm{~d} r\right) .
\end{aligned}
$$

It then suffices to take $k, \rho_{1}$ and $\rho_{2}$ s.t. the quantities $\rho_{i}^{2} k, i=1,2$, are small enough uniformly.

Let us now consider the contribution of the first jump term. Clearly by Lemma 1.3 we have for some $c>0$, all $\epsilon>0$, all $k \geq 1$ and all jump times $t_{i}^{k}$

$$
\left|\Delta \widehat{Y}_{t_{i}^{k}}^{k, \epsilon}\right| \leq c \epsilon\left|\Delta_{t_{i-1}^{k}, t_{i}^{k}} Y^{\epsilon}\right| .
$$


There exist two constants $c(k), c^{\prime}(k)>0$ and an $\epsilon_{0}>0$ s.t. for all $\epsilon \leq \epsilon_{0}$ we have by equation (1.10) and Lemma 2.7

$$
\begin{aligned}
E\left|\widehat{Y}_{s^{*}(k)-}^{k, \epsilon}\right|\left|\Delta \widehat{Y}_{s^{*}(k)}^{k, \epsilon}\right| & \leq c\left(\epsilon E\left|\widehat{Y}_{s^{*}(k)}^{k, \epsilon}-\right|^{2}+\frac{1}{\epsilon} E\left|\Delta \widehat{Y}_{s^{*}(k)}^{k, \epsilon}\right|^{2}\right) \\
& \leq c \epsilon\left(\sup _{s \leq r \leq T} E\left|\widehat{Y}_{r}^{k, \epsilon}\right|^{2}+E\left|\Delta_{s_{*(k)}, s^{*(k)}} Y_{.}^{\epsilon}\right|^{2}\right) \\
& \leq c+c^{\prime} \epsilon \sup _{s \leq r \leq T} E\left|\widehat{Y}_{r}^{k, \epsilon}\right|^{2} .
\end{aligned}
$$

Let us at last consider the remaining jump terms. We have for any number $\nu$ in $(0,1)$ by our interpolation equation (1.10)

$$
\begin{aligned}
E \sum_{t_{i}^{k}>s^{*(k)}}^{T_{*(k)}}\left|\widehat{Y}_{t_{i}^{k}-}^{k, \epsilon}\right|\left|\Delta \widehat{Y}_{t_{i}^{k}}^{k, \epsilon}\right| & \leq c\left[k^{\nu}\left(\sum_{t_{i}^{k}>s^{*(k)}}^{T_{*(k)}} \epsilon^{2} E\left|\widehat{Y}_{t_{i}^{k}-\epsilon}^{k, \epsilon}\right|^{2}\right)+k^{-\nu}\left(\sum_{t_{i}^{k}>s^{*(k)}}^{T_{*(k)}} E\left|\Delta_{t_{i-1}^{k}, t_{i}^{k}} Y^{\epsilon}\right|^{2}\right)\right] \\
& \leq c\left[k^{\nu}\left(\sup _{s \leq r \leq T} E\left|\widehat{Y}_{r}^{k, \epsilon}\right|^{2} \sum_{t_{i}^{k}>s^{*(k)}}^{T_{*(k)}} \epsilon^{2}\right)+k^{-\nu}\left(\sum_{t_{i}^{k}>s^{*(k)}}^{T_{*(k)}} E\left|\Delta_{t_{i-1}^{k}, t_{i}^{k}} Y^{\epsilon}\right|^{2}\right)\right] \\
& \leq c\left(k^{\nu-1} \sup _{s \leq r \leq T} E\left|\widehat{Y}_{r}^{k, \epsilon}\right|^{2}+k^{-\nu} \sum_{t_{i}^{k}>s^{*(k)}}^{T_{*(k)}} E\left|\Delta_{t_{i-1}^{k}, t_{i}^{k}} Y_{.}^{\epsilon}\right|^{2}\right) .
\end{aligned}
$$

The sum on the right hand-side above is treated as in the inequality (2.11) (there is however no $k$ in front). The remaining terms are easy.

Now, adding all these terms together, we find that for some constant $c=c\left(\rho_{1}, \rho_{2}, k\right)>0$, there is an $\epsilon_{0}>0$ s.t. for all $\epsilon \leq \epsilon_{0}$ and all $s$ in $[0, T]$

$$
\sup _{s \leq r \leq T} E\left|\widehat{Y}_{r}^{k, \epsilon}\right|^{2}+E \int_{s}^{T}\left\|\widehat{Z}_{r}^{k, \epsilon}\right\|^{2} \mathrm{~d} r \leq c\left(1+E\left|g\left(X_{T}^{\epsilon}\right)\right|^{2}+\int_{s}^{T} \sup _{r \leq u \leq T} E\left|\widehat{Y}_{u}^{k, \epsilon}\right|^{2} \mathrm{~d} r\right) .
$$

We are now in the position to establish our sought-for estimate.

Lemma 2.9. Under the above notations, there exists a $c>0$ which depends only on $d, T$ and other absolute constants from condition $A$ and an $\epsilon_{0}>0$ s.t. when $\epsilon \leq \epsilon_{0}$, we have

$$
E\left(\sup _{0 \leq s \leq T}\left|\widehat{Y}_{s}^{k, \epsilon}\right|^{2}\right)+E \int_{0}^{T}\left\|\widehat{Z}_{r}^{k, \epsilon}\right\|^{2} \mathrm{~d} r<c .
$$

Proof. It follows from the fact that $g$ is bounded and from using the Gronwall-Bellman inequality on the inequality (2.12) that the following inequality holds for some $c>0$, for some fixed $k$, all $\epsilon$ sufficiently small and all $s$ in $[0, T]$,

$$
\sup _{s \leq r \leq T} E\left|\widehat{Y}_{r}^{k, \epsilon}\right|^{2}+E \int_{s}^{T}\left\|\widehat{Z}_{r}^{k, \epsilon}\right\|^{2} \mathrm{~d} r \leq c .
$$

A further application of the Burkholder-Davis-Gundy inequality on the Itô formula (2.10) above gives the result. 
Now we go back to our original process $Y^{\epsilon}$. We can state the

Corollary 2.10. Under the above notations, there exists a $c>0$ which depends only on $d, T$ and other absolute constants from condition $A$ and an $\epsilon_{0}>0$ s.t. when $\epsilon \leq \epsilon_{0}$, we have

$$
\sup _{\epsilon \leq \epsilon_{0}}\left(E \sup _{0 \leq s \leq T}\left|Y_{s}^{\epsilon}\right|^{2}+E \int_{0}^{T}\left\|Z_{r}^{\epsilon}\right\|^{2} \mathrm{~d} r\right)<c .
$$

Proof. Let us just remark that the use of Lemmas 1.3, 2.2 and the relation (2.8) in the above bounds yields that there exists a $c>0$ s.t. for all $\epsilon$ sufficiently small, we have

$$
E\left(\sup _{0 \leq s \leq T}\left|Y_{s}^{\epsilon}\right|^{2}\right)+E \int_{0}^{T}\left\|Z_{r}^{\epsilon}\right\|^{2} \mathrm{~d} r<c(k) .
$$

In particular from the estimate

$$
\sup _{0 \leq s \leq T} E\left(\left|Y_{s}^{\epsilon, 0, x_{0}}\right|^{2}\right)<c(k),
$$

we deduce thanks to the fact that $Y_{0}^{\epsilon, 0, x_{0}}$ is deterministic, to the fact that $g$ is bounded and from Theorems 3.1 and 3.2 (existence and uniqueness results which imply the Markovian character of the process $Y_{s}^{\epsilon}$ for any $\epsilon>0$ ) of Delarue[7] that there exists a constant $c_{y}$, which depends only on $d, T$ and other absolute constants that appear in Condition A, s.t. for any $\epsilon \leq 1$

$$
P\left(\sup _{0 \leq s \leq T}\left|Y_{s}^{\epsilon}\right| \leq c_{y}\right)=1
$$

and consequently we also have by Lemma 1.3 , for some constant $\widehat{c}_{y}$ and $\epsilon \leq 1$

$$
P\left(\sup _{0 \leq s \leq T}\left|\widehat{Y}_{s}^{k, \epsilon}\right| \leq \widehat{c}_{y}\right)=1 .
$$

We can also deduce the following from our fundamental estimate above,

Lemma 2.11. There exists a constant $c_{z}$ s.t. for all $\epsilon$ sufficiently small

$$
E\left(\int_{0}^{T}\left\|Z_{r}^{\epsilon}\right\|^{2} \mathrm{~d} r\right)^{2}<c_{z}
$$

Proof. The first step consists in writing thanks to the Itô formula (2.10) the inequality

$$
\begin{aligned}
E\left(\sup _{0 \leq s \leq T}\left|\widehat{Y}_{s}^{k, \epsilon}\right|^{4}\right)+E\left(\int_{0}^{T}\left\|\widehat{Z}_{r}^{k, \epsilon}\right\|^{2} \mathrm{~d} r\right)^{2} \leq & c\left[E\left|g\left(X_{T}^{\epsilon}\right)-\epsilon \widehat{e}\left(T, T, Y_{T_{*(k)}}^{\epsilon}\right)\right|^{4}+E\left|\int_{0}^{T} \widehat{Y}_{r}^{k, \epsilon} \widehat{U}_{r}^{k, \epsilon} \mathrm{d} r\right|^{2}\right. \\
& \left.+E\left(\sup _{0 \leq s \leq T}\left|\widehat{Y}_{s}^{k, \epsilon}\right|^{2} \int_{0}^{T}\left\|\widehat{Z}_{r}^{k, \epsilon}\right\|^{2} \mathrm{~d} r\right)+E\left|\sum_{t_{i}^{k}>0}^{T_{*(k)}} \widehat{Y}_{t_{i}^{k}-}^{k, \epsilon} \cdot \Delta \widehat{Y}_{t_{i}^{k}}^{k, \epsilon}\right|^{2}\right] .
\end{aligned}
$$


Using the above computations and the equality (2.15) we can show that for some absolute constant $c_{z}$, only depending on absolute constants from Condition A, we have for all $0<\epsilon<1$,

$$
E\left(\int_{0}^{T}\left\|Z_{r}^{\epsilon}\right\|^{2} \mathrm{~d} r\right)^{2}<c_{z} .
$$

Recall again that the backward process is given by

$$
Y_{s}^{\epsilon}=g\left(X_{T}^{\epsilon}\right)+\int_{s}^{T}\left(\frac{1}{\epsilon} e+f\right)\left(\frac{X_{r}^{\epsilon}}{\epsilon}, X_{r}^{\epsilon}, Y_{r}^{\epsilon}, Z_{r}^{\epsilon}\right) \mathrm{d} r-\int_{s}^{T} Z_{r}^{\epsilon} \mathrm{d} B_{r}
$$

Let us now set

$$
\widehat{Y}_{s}^{\epsilon}=Y_{s}^{\epsilon}-\epsilon \widehat{e}\left(\frac{X_{s}^{\epsilon}}{\epsilon}, X_{s}^{\epsilon}, Y_{s}^{\epsilon}\right)
$$

and write thanks again to the Itô formula

$$
\begin{aligned}
\widehat{Y}_{s}^{\epsilon}= & \widehat{Y}_{T}^{\epsilon}+\int_{s}^{T}\left\{\left(\partial_{x^{1}} \widehat{e} b^{\prime}+\partial_{x^{2}} \widehat{e} b-\partial_{y} \widehat{e} e+\operatorname{Tr}_{x^{1} x^{2}}^{2} \widehat{e} a+\partial_{x^{1}} \widehat{e} a \partial_{x^{1} y}^{2} \widehat{e}\right.\right. \\
& +f)\left(r, r, Y_{r}^{\epsilon}, \widetilde{Z}_{r}^{\epsilon}+\partial_{x^{1}} \widehat{e} \sigma\right)+\epsilon\left[\partial_{x^{2}} \widehat{e} b^{\prime}\left(r, r, Y_{r}^{\epsilon}\right)-\partial_{y} \widehat{e} f\left(r, r, Y_{r}^{\epsilon}, Z_{r}^{\epsilon}\right)\right. \\
& +\partial_{x^{2}} \widehat{e} a \partial_{x^{1} y}^{2} \widehat{e}\left(r, r, Y_{r}^{\epsilon}\right)+\partial_{y} \widehat{e}\left(r, r, Y_{r}^{\epsilon}\right) Z_{r}^{\epsilon} \sigma^{*} \partial_{x^{1} y}^{2} \widehat{e}\left(r, r, Y_{r}^{\epsilon}\right) \\
& \left.\left.+\frac{1}{2} \operatorname{Tr}_{x^{2}}^{2} \widehat{e} a\left(r, r, Y_{r}^{\epsilon}\right)+\partial_{x^{2} y}^{2} \widehat{e} \sigma\left(r, r, Y_{r}^{\epsilon}\right) Z_{r}^{\epsilon}+\frac{1}{2} \partial_{y}^{2} \widehat{e}\left(r, r, Y_{r}^{\epsilon}\right)\left\|Z_{r}^{\epsilon}\right\|^{2}\right]\right\} \mathrm{d} r \\
& -\int_{s}^{T}\left\{\widetilde{Z}_{r}^{\epsilon}-\epsilon\left[\partial_{x^{2}} \widehat{e} \sigma\left(r, r, Y_{r}^{\epsilon}\right)+\partial_{y} \widehat{e}\left(r, r, Y_{r}^{\epsilon}\right) Z_{r}^{\epsilon}\right]\right\} \mathrm{d} \widetilde{B}_{r},
\end{aligned}
$$

where

$$
\begin{aligned}
\widetilde{B}_{r} & =B_{r}-\int_{0}^{r} \sigma^{*} \partial_{x^{1} y}^{2} \widehat{e}\left(u, u, Y_{u}^{\epsilon}\right) \mathrm{d} u \\
\widetilde{Z}_{r}^{\epsilon} & =Z_{r}^{\epsilon}-\left(\partial_{x^{1}} \widehat{e} \sigma\right)\left(r, r, Y_{r}^{\epsilon}\right) .
\end{aligned}
$$

We define thanks to a Girsanov's transform a new probability measure $\widetilde{P}$ s.t. under $\widetilde{P}$ the process $\widetilde{B}_{r}$ is a Brownian motion. Define also the martingale

$$
M_{s}^{\epsilon}=-\int_{0}^{s} \widetilde{Z}_{r}^{\epsilon} \mathrm{d} \widetilde{B}_{r}
$$

Note that from the equality $(2.14)$

$$
\widetilde{P}\left(\sup _{0 \leq s \leq T}\left|Y_{s}^{\epsilon}\right| \leq c_{y}\right)=1
$$

We are now in the position to state the

Corollary 2.12. The family of processes $\left(Y^{\epsilon}, M^{\epsilon}\right)$ in $[0, T]$ indexed by $\epsilon$ is $\widetilde{P}$-tight in the Jakubowski $S$ topology. Proof. The following criterion ensures tightness in the Jakubowski $S$-topology for a càdlàg family of processes $y_{s}^{\epsilon}$

$$
\sup _{\epsilon \leq \epsilon_{0}}\left[\sup _{s \leq T} \widetilde{E}\left|y_{s}^{\epsilon}\right|+C V(T)\right]<\infty,
$$


where $C V(T)$ stands for the conditional variation

$$
C V(T)=\sup \left\{\widetilde{E} \sum_{i}\left|\widetilde{E}\left(\Delta_{t_{i-1}, t_{i}} y_{.}^{\epsilon} \mid \mathcal{F}_{t_{i-1}}\right)\right|\right\},
$$

where the supremum is taken over all finite partitions of the interval $[0, T]$. We first show that the process $y_{s}^{\epsilon}=\widehat{Y}_{s}^{\epsilon}$ is $P$-tight. Since $\epsilon \widehat{e}\left(\frac{X_{s}^{\epsilon}}{\epsilon}, X_{s}^{\epsilon}, Y_{s}^{\epsilon}\right)$ converges to zero (in sup-norm) a.s. by the relation (2.18), tightness for the process $Y_{s}^{\epsilon}$ itself follows from that of $\widehat{Y}_{s}^{\epsilon}$. The tightness for the martingale $M_{s}^{\epsilon}$ is clearly established in the same way. Let $t_{i}$ be any partition of $[0, T]$. From Lemmas 1.3, 2.11, 1.2 and from the relations (2.13) and (2.18) the first term on the left hand-side of the formula (2.19) is easily dealt with. It remains only to note that by the elementary properties of the conditional expectation we have

$$
\begin{aligned}
\widetilde{E} \sum_{i}\left|\widetilde{E}\left(\Delta_{t_{i-1}, t_{i}} y_{.}^{\epsilon} \mid \mathcal{F}_{t_{i}}\right)\right| & \leq c \widetilde{E} \sum_{i} \int_{t_{i-1}}^{t_{i}}\left(1+\left|Y_{r}^{\epsilon}\right|+\left\|Z_{r}^{\epsilon}\right\|+\epsilon\left\|Z_{r}^{\epsilon}\right\|^{2}\right) \mathrm{d} r \\
& \leq c \widetilde{E} \int_{0}^{T}\left(1+\left\|Z_{r}^{\epsilon}\right\|+\epsilon\left\|Z_{r}^{\epsilon}\right\|^{2}\right) \mathrm{d} r .
\end{aligned}
$$

Our corollary follows immediately from Lemma 1.2, Corollary 2.10, Lemma 2.11 and the Cauchy-Schwarz inequality.

Remark 2.13. Note that once an appropriate $k$ is chosen it is kept fixed and that $c(k) \rightarrow \infty$ as $k \rightarrow \infty$. It is also important to notice that the constant on the right-hand side of the inequality (2.13) depends only on absolute constants from Condition A and on bounds on $E\left[g\left(X_{T}^{\epsilon}\right)\right]^{2}$.

\section{The CONVERGence $u^{\epsilon}(t, x) \rightarrow u(t, x)$}

Fix $0 \leq t \leq T$ and $x$ in $R^{d}$. All our processes below are running on the time interval $[t, T]$ and $X_{s}^{\epsilon}$ starts from $x$, but most of the time we omit these superscripts. Recall that the backward process is given by

$$
Y_{s}^{\epsilon}=g\left(X_{T}^{\epsilon}\right)+\int_{s}^{T}\left(\frac{1}{\epsilon} e+f\right)\left(r, r, Y_{r}^{\epsilon}, Z_{r}^{\epsilon}\right) \mathrm{d} r-\int_{s}^{T} Z_{r}^{\epsilon} \mathrm{d} B_{r}
$$

Let us now rewrite the formula (2.16),

$$
\begin{aligned}
Y_{s}^{\epsilon}= & g\left(X_{T}^{\epsilon}\right)+\int_{s}^{T}\left(\partial_{x^{1}} \widehat{e} b^{\prime}+\partial_{x^{2}} \widehat{e} b-\partial_{y} \widehat{e} e+\operatorname{Tr}_{x^{1} x^{2}}^{2} \widehat{e} a+\partial_{x^{1}} \widehat{e} a \partial_{x^{1} y}^{2} \widehat{e}\right. \\
& +f)\left(r, r, Y_{r}^{\epsilon}, \widetilde{Z}_{r}^{\epsilon}+\partial_{x^{1}} \widehat{e} \sigma\right) \mathrm{d} r-\int_{s}^{T} \widetilde{Z}_{r}^{\epsilon} \mathrm{d} \widetilde{B}_{r}+\rho_{s}^{\epsilon}
\end{aligned}
$$

where it is recalled that

$$
\begin{aligned}
\widetilde{B}_{r} & =B_{r}-\int_{0}^{r} \sigma^{*} \partial_{x^{1} y}^{2} \widehat{e}\left(u, u, Y_{u}^{\epsilon}\right) \mathrm{d} u \\
\widetilde{Z}_{r}^{\epsilon} & =Z_{r}^{\epsilon}-\left(\partial_{x^{1}} \widehat{e} \sigma\right)\left(r, r, Y_{r}^{\epsilon}\right)
\end{aligned}
$$

and

$$
\rho_{s}^{\epsilon}=\rho_{1, s}^{\epsilon}+\rho_{2, s}^{\epsilon}
$$


where

$$
\begin{aligned}
\rho_{1, s}^{\epsilon}= & \epsilon \int_{s}^{T}\left[\partial_{x^{2}} \widehat{e} b^{\prime}\left(r, r, Y_{r}^{\epsilon}\right)-\partial_{y} \widehat{e} f\left(r, r, Y_{r}^{\epsilon}, Z_{r}^{\epsilon}\right)+\partial_{x^{2}} \widehat{e} a \partial_{x^{1} y}^{2} \widehat{e}\left(r, r, Y_{r}^{\epsilon}\right)\right. \\
& +\partial_{y} \widehat{e}\left(r, r, Y_{r}^{\epsilon}\right) Z_{r}^{\epsilon} \sigma^{*} \partial_{x^{1} y}^{2} \widehat{e}\left(r, r, Y_{r}^{\epsilon}\right) \\
& \left.+\frac{1}{2} \operatorname{Tr} \partial_{x^{2}}^{2} \widehat{e} a\left(r, r, Y_{r}^{\epsilon}\right)+\partial_{x^{2} y}^{2} \widehat{e} \sigma\left(r, r, Y_{r}^{\epsilon}\right) Z_{r}^{\epsilon}+\frac{1}{2} \partial_{y}^{2} \widehat{e}\left(r, r, Y_{r}^{\epsilon}\right)\left\|Z_{r}^{\epsilon}\right\|^{2}\right] \mathrm{d} r \\
& +\epsilon \int_{s}^{T}\left[\partial_{x^{2}} \widehat{e} \sigma\left(r, r, Y_{r}^{\epsilon}\right)+\partial_{y} \widehat{e}\left(r, r, Y_{r}^{\epsilon}\right) Z_{r}^{\epsilon}\right] \mathrm{d} \widetilde{B}_{r},
\end{aligned}
$$

and

$$
\rho_{2, s}^{\epsilon}=\epsilon\left[\widehat{e}\left(s, s, Y_{s}^{\epsilon}\right)-\widehat{e}\left(T, T, Y_{T}^{\epsilon}\right)\right] .
$$

The change of measure from $P$ to $\widetilde{P}$ we made while studying tightness has also consequences on the forward process. That is, the processes $\bar{X}_{s}^{\epsilon}$ and $R_{s}^{\epsilon}$ defined in (2.2) undergo changes similar to those in $Y_{s}^{\epsilon}$ and $\rho_{s}^{\epsilon}$, we only notice that

$$
\bar{X}_{s}^{\epsilon}=x+\int_{t}^{s}\left(F^{\prime}+G \sigma^{*} \partial_{x^{1} y}^{2} \widehat{e}\right)\left(\frac{X_{r}^{\epsilon}}{\epsilon}, X_{r}^{\epsilon}, Y_{r}^{\epsilon}\right) \mathrm{d} r+\int_{t}^{s} G\left(\frac{X_{r}^{\epsilon}}{\epsilon}, X_{r}^{\epsilon}\right) \mathrm{d} \widetilde{B}_{r} .
$$

Define in this respect

$$
\begin{aligned}
F & =F^{\prime}+G \sigma^{*} \partial_{x^{1} y}^{2} \widehat{e} \\
\Lambda & =G G^{*}
\end{aligned}
$$

Recall also that we proved in the previous section while studying tightness that there exists a constant $c_{y}$, which depends only on $d, T$ and other absolute constants that appear in Condition A, and an $\epsilon_{0}>0$ s.t. for any $\epsilon \leq \epsilon_{0}$

$$
P\left(\sup _{0 \leq s \leq T}\left|Y_{s}^{\epsilon}\right| \leq c_{y}\right)=1 .
$$

Concerning the $Z^{\epsilon}$ process, we will also need an eighth power in what follows.

Lemma 3.1. There exists an $\epsilon_{0}>0$ and a constant $c_{z}$ s.t. for all $0<\epsilon \leq \epsilon_{0}$

$$
E\left[\left(\int_{0}^{T}\left\|Z_{r}^{\epsilon}\right\|^{2} \mathrm{~d} r\right)^{4}\right]<c_{z} .
$$

Proof. It suffices to take the fourth power in equation (2.10) and to use the estimate of Lemma 2.11 together with that of equation (2.15).

The two latter facts together with the relation (2.3) yield, thanks to uniform integrability and to the boundedness of the integrands, the

Corollary 3.2. For each $(t, x)$ in $[0, T] \times R^{d}$, the following convergences

$$
\sup _{s \leq T}\left\|R_{s}^{\epsilon}\right\| \rightarrow 0
$$

and

hold in $L^{2}(\widetilde{P})$ as $\epsilon \rightarrow 0$.

$$
\sup _{s \leq T}\left|\rho_{s}^{\epsilon}\right| \rightarrow 0
$$


By the above considerations and by Lemma 1.2 , the sequence of processes $\left(X_{.}^{\epsilon}, Y^{\epsilon}, M^{\epsilon}\right)$ is $\widetilde{P}$-tight in $\mathcal{C}([0, T]) \times(\mathcal{D}[0, T])^{2}$ where the first space is equipped with the sup-norm and the two other factors with the Jakubowski $S$-topoloy.

The main nonlinear coefficient in the backward process is given by

$$
U\left(x^{1}, x^{2}, y, z\right)=\left(\partial_{x^{1}} \widehat{e} b^{\prime}+\partial_{x^{2}} \widehat{e} b-\partial_{y} \widehat{e} e+\operatorname{Tr}_{x^{1} x^{2}}^{2} \widehat{e} a+\partial_{x^{1}} \widehat{e} a \partial_{x^{1} y}^{2} \widehat{e}+f\right)\left(x^{1}, x^{2}, y, z+\partial_{x^{1}} \widehat{e} \sigma\right),
$$

and therefore the process $Y_{s}^{\epsilon}$ has the same asymptotic behaviour as the process

$$
\bar{Y}_{s}^{\epsilon}=g\left(X_{T}^{\epsilon}\right)+\int_{s}^{T} U\left(\frac{X_{r}^{\epsilon}}{\epsilon}, X_{r}^{\epsilon}, Y_{r}^{\epsilon}, \widetilde{Z}_{r}^{\epsilon}\right) \mathrm{d} r-\int_{s}^{T} \widetilde{Z}_{r}^{\epsilon} \mathrm{d} \widetilde{B}_{r}
$$

Note that we have

$$
U\left(x^{1}, x^{2}, y, z\right)=U_{1}\left(x^{1}, x^{2}, y\right)+U_{2}\left(x^{1}, x^{2}, y, z\right)
$$

where $U_{1}$ is the part of $U$ that does not depend on $z$ and

$$
U_{2}\left(x^{1}, x^{2}, y, z\right)=f\left(x^{1}, x^{2}, y, z+\partial_{x^{1}} \widehat{e} \sigma\left(x^{1}, x^{2}, y\right)\right) .
$$

Define also the martingale

$$
M_{s}^{\epsilon}=-\int_{0}^{s} \widetilde{Z}_{r}^{\epsilon} \mathrm{d} \widetilde{B}_{r}
$$

Since the gradient $\nabla u^{\epsilon}(t, x)$ is not sufficiently well behaved as $\epsilon \rightarrow 0$, we are not going to identify the limit points of the family $\left(X^{\epsilon}, Y_{.}^{\epsilon}, M^{\epsilon}\right)$ by the usual weak-limit methods, instead we only prove that $u^{\epsilon}(t, x) \rightarrow u(t, x)$. Let us homogenize our coefficients to see the limit operator

$$
\begin{aligned}
\bar{F}\left(x^{2}, y\right) & =\int_{\mathbb{T}^{d}} F\left(x^{1}, x^{2}, y\right) \mu\left(\mathrm{d} x^{1}, x^{2}\right), \\
\bar{\Lambda}\left(x^{2}\right) & =\int_{\mathbb{T}^{d}} \Lambda\left(x^{1}, x^{2}\right) \mu\left(\mathrm{d} x^{1}, x^{2}\right), \\
\bar{U}\left(x^{2}, y, z\right) & =\int_{\mathbb{T}^{d}} U\left[x^{1}, x^{2}, y, z G\left(x^{1}, x^{2}\right)+\partial_{x^{1}} \widehat{e} \sigma\left(x^{1}, x^{2}, y\right)\right] \mu\left(\mathrm{d} x^{1}, x^{2}\right) .
\end{aligned}
$$

Consider now the non-linear parabolic PDE

$$
\left\{\begin{aligned}
\partial_{t} u(t, x)+\Gamma\left(x, u(t, x), \nabla u(t, x), \partial^{2} u(t, x)\right) & =0, \\
u(T, x) & =g(x),
\end{aligned}\right.
$$

where

in which

$$
\Gamma\left(x, u(t, x), \nabla u(t, x), \partial^{2} u(t, x)\right)=\bar{L} u(t, x)+\bar{U}(x, u(t, x), \nabla u(t, x)),
$$

$$
\bar{L}(x, .)=\frac{1}{2} \sum_{i, j=1}^{d} \bar{\Lambda}_{i j}(x) \partial_{x_{i} x_{j}}^{2}(.)+\sum_{i=1}^{d} \bar{F}_{i}(x, .) \partial_{x_{i}}(.),
$$

and $\bar{U}$ is given by (3.5). The fact that our limit PDE is strongly non degenerate, i.e.

$$
(\bar{\Lambda}(x) \xi, \xi) \geq \bar{\lambda}\|\xi\|^{2},
$$

for some $\bar{\lambda}>0$ and all $(\xi, x)$ in $R^{2 d}$, follows easily from the relations (3.5) and from the well known bounds on the density $p_{\infty}\left(x^{1}, x^{2}\right)$ of the invariant measure which are uniform in the variables $\left(x^{1}, x^{2}\right)$. It also follows from Condition A and Lemma 1.3 that the nonlinear coefficient

$$
\Gamma^{\prime}(x, y, z)=(\bar{F}(x, y), z)+\bar{U}(x, y, z),
$$


is locally Lipschitz continuous with

$$
\begin{aligned}
\left\|\partial_{x} \Gamma^{\prime}(x, y, z)\right\| & \leq c(1+|y|+\|z\|), \\
\left|\partial_{y} \Gamma^{\prime}(x, y, z)\right| & \leq c, \\
\left\|\partial_{z} \Gamma^{\prime}(x, y, z)\right\| & \leq c,
\end{aligned}
$$

for some constant $c$ and all $(x, y, z)$ in $R^{2 d+1}$. Theorem 6.1 of [7] implies that the semi-linear PDE (3.6) has a unique bounded continuous viscosity solution $u(t, x)$ in $\mathcal{C}^{1,2}\left([0, T) \times R^{d}, R\right) \cap \mathcal{C}\left([0, T] \times R^{d}, R\right)$ s.t.

$$
\left|u\left(t_{1}, x_{1}\right)-u\left(t_{2}, x_{2}\right)\right| \leq c\left(\left|t_{2}-t_{1}\right|^{1 / 2}+\left\|x_{2}-x_{1}\right\|\right)
$$

while the gradient $\nabla u(s, x)$ is $\beta$-Holder continuous, for some $\beta \in(0,1]$, in $[t, T-\delta] \times R^{d}$ for any $\delta \in(0, T-t)$. We need however more regularity in what follows.

\subsection{Smoothing of some coefficients}

We shall now regularize the functions $g$ and $\bar{U}_{2}$ (recall that $\bar{U}_{2}$ is defined in (3.3) and (3.5)) in such a way that the solution of the PDE

$$
\left\{\begin{aligned}
\partial_{t} \bar{u}^{\epsilon}(t, x)+\Gamma^{\epsilon}\left(x, \bar{u}^{\epsilon}(t, x), \nabla \bar{u}^{\epsilon}(t, x), \partial^{2} \bar{u}^{\epsilon}(t, x)\right) & =0, \\
\bar{u}^{\epsilon}(T, x) & =g^{\epsilon}(x),
\end{aligned}\right.
$$

with

$$
\Gamma^{\epsilon}\left(x, \bar{u}^{\epsilon}(t, x), \nabla \bar{u}^{\epsilon}(t, x), \partial^{2} \bar{u}^{\epsilon}(t, x)\right)=\bar{L}_{\bar{u}}^{\epsilon}(t, x)+\bar{U}_{1}\left(x, \bar{u}^{\epsilon}(t, x)\right)+\bar{U}_{2}^{\epsilon}\left(x, \bar{u}^{\epsilon}(t, x), \nabla \bar{u}^{\epsilon}(t, x)\right),
$$

has a bounded Hessian matrix.

In order to explain this smoothing which depends on $\epsilon$ itself, let $\theta_{1}(\epsilon)$ and $\theta_{2}(\epsilon)$ be two positive functions of $\epsilon>0$ that tend to zero as $\epsilon \rightarrow 0$. They will be fixed below. Now consider the regularized coefficients

$$
\begin{aligned}
g^{\epsilon}(x) & =\theta_{1}^{-d} \int_{R^{d}} g\left(x-x^{\prime}\right) \varphi\left(\frac{x^{\prime}}{\theta_{1}}\right) \mathrm{d} x^{\prime} \\
\bar{U}_{2}^{\epsilon}(x, y, z) & =\theta_{2}^{-(2 d+1)} \int_{R^{2 d+1}} \bar{U}_{2}\left(x-x^{\prime}, y-y^{\prime}, z-z^{\prime}\right) \varphi\left(\frac{x^{\prime}}{\theta_{2}}, \frac{y^{\prime}}{\theta_{2}}, \frac{z^{\prime}}{\theta_{2}}\right) \mathrm{d} x^{\prime} \mathrm{d} y^{\prime} \mathrm{d} z^{\prime},
\end{aligned}
$$

where the mollifier $\varphi$ is a smooth function with compact support in $R^{2 d+1}$. These regularized functions $\bar{U}_{2}^{\epsilon}$ and $g^{\epsilon}$ remain (locally) Lipschitz with their former constants. It follows from [8] p. 2322 that the solution $\bar{u}^{\epsilon}(t, x)$ of the Cauchy problem (3.9) satisfies for some constants $c_{\nabla}>0, M(\epsilon)>0$ and all $(t, x) \in[0, T] \times R^{d}$

$$
\begin{aligned}
\sup _{\epsilon>0}\left(\left|\bar{u}^{\epsilon}(t, x)\right|+\left\|\nabla \bar{u}^{\epsilon}(t, x)\right\|\right) & \leq c_{\nabla}, \\
\left\|\partial_{x}^{2} \bar{u}^{\epsilon}(t, x)\right\| & \leq M(\epsilon) ;
\end{aligned}
$$

for all $\epsilon>0$, where $c_{\nabla}$ is a constant that depends only on absolute constants from Condition $\mathrm{A}$ and $M(\epsilon)$ depends on $\theta_{1}(\epsilon)$ and $\theta_{2}(\epsilon)$ with $M(\epsilon) \rightarrow \infty$ as $\epsilon \rightarrow 0$ when we are dealing with "true" viscosity solutions with unbounded second derivatives defined on compacts of $[0, T) \times R^{d}$; which is our standpoint in this paper. On the other hand, we also have for any $\beta \in(0,1), \bar{u}^{\epsilon} \in \mathcal{C}^{(1+\beta / 2,2+\beta)}\left([0, T] \times R^{d}, R\right)$, i.e. in particular for all $\left(t_{1}, x_{1}\right)$ and $\left(t_{2}, x_{2}\right)$ in $[t, T] \times R^{d}$,

$$
\left\|\partial_{x}^{2} \bar{u}^{\epsilon}\left(t_{1}, x_{1}\right)-\partial_{x}^{2} \bar{u}^{\epsilon}\left(t_{2}, x_{2}\right)\right\| \leq N(\epsilon)\left(\left|t_{2}-t_{1}\right|^{\beta / 2}+\left\|x_{2}-x_{1}\right\|^{\beta}\right),
$$

where $N(\epsilon) \rightarrow \infty$ as $\epsilon \rightarrow 0$. 
The following obvious technical result will be needed below. Fix a $\beta \in(0,1)$; then it is possible to choose $\theta_{1}(\epsilon)$ and $\theta_{2}(\epsilon)$ sufficiently large compared to $\epsilon$ s.t. as $\epsilon \rightarrow 0$ we have

$$
\begin{aligned}
\epsilon M(\epsilon) & \rightarrow 0, \\
\epsilon^{\beta} N(\epsilon) & \rightarrow 0 .
\end{aligned}
$$

We also need the following crucial lemma the proof of which is deferred to Section 4.

Lemma 3.3. With the above notations, for all $(t, x) \in[0, T] \times R^{d}$,

$$
u^{\epsilon}(t, x)-\bar{u}^{\epsilon}(t, x) \rightarrow 0
$$

as $\epsilon \rightarrow 0$.

\subsection{Identification of the limit}

Our main result in this paper is the following

Theorem 3.4. Under condition A, there exists a unique bounded continuous viscosity solution $u(t, x)$ of equation (3.6). For any $(t, x) \in[0, T] \times R^{d}$,

as $\epsilon \rightarrow 0$.

$$
u^{\epsilon}(t, x) \rightarrow u(t, x)
$$

Proof. Fix $(t, x) \in[0, T] \times R^{d}$. It follows from Remark 6.3 p. 34 of [6] that

$$
\bar{u}^{\epsilon}(t, x) \rightarrow u(t, x)
$$

as $\epsilon \rightarrow 0$. Hence the Theorem follows from Lemma 3.3.

\section{Proof of Lemma 3.3}

Fix $(t, x) \in[0, T] \times R^{d}$. Recall that we have

$$
\begin{aligned}
& \bar{X}_{s}^{\epsilon, t, x}=x+\int_{t}^{s} F\left(\frac{X_{r}^{\epsilon, t, x}}{\epsilon}, X_{r}^{\epsilon, t, x}, Y_{r}^{\epsilon, t, x}\right) \mathrm{d} r+\int_{t}^{s} G\left(\frac{X_{r}^{\epsilon, t, x}}{\epsilon}, X_{r}^{\epsilon, t, x}\right) \mathrm{d} \widetilde{B}_{r}, \\
& \bar{Y}_{s}^{\epsilon, t, x}=g\left(X_{T}^{\epsilon, t, x}\right)+\int_{s}^{T} U\left(\frac{X_{r}^{\epsilon, t, x}}{\epsilon}, X_{r}^{\epsilon, t, x}, Y_{r}^{\epsilon, t, x}, \widetilde{Z}_{r}^{\epsilon, t, x}\right) \mathrm{d} r-\int_{s}^{T} \widetilde{Z}_{r}^{\epsilon, t, x} \mathrm{~d} \widetilde{B}_{r} .
\end{aligned}
$$

We note that

$$
\left|u^{\epsilon}(t, x)-\bar{u}^{\epsilon}(t, x)\right| \leq \widetilde{E}\left|Y_{t}^{\epsilon, t, x}-\bar{Y}_{t}^{\epsilon, t, x}\right|+\widetilde{E}\left|\bar{Y}_{t}^{\epsilon, t, x}-\bar{u}^{\epsilon}(t, x)\right|
$$

From Corollary 3.2 we have

$$
\begin{aligned}
\widetilde{E}\left|Y_{t}^{\epsilon, t, x}-\bar{Y}_{t}^{\epsilon, t, x}\right| & =\widetilde{E}\left|\rho_{t}^{\epsilon}\right| \\
& \rightarrow 0
\end{aligned}
$$

as $\epsilon \rightarrow 0$. Let us deal with the second term on the right hand-side of the above inequality. We can write (from now on, $\bar{r}$ stands for $\bar{X}_{r}^{\epsilon}, \bar{s}$ for $\bar{X}_{s}^{\epsilon}$ and $\bar{T}$ for $\bar{X}_{T}^{\epsilon}$ )

$$
\begin{aligned}
\bar{Y}_{s}^{\epsilon}= & g\left(X_{T}^{\epsilon}\right)+\int_{s}^{T}\left[U_{2}\left(r, r, Y_{r}^{\epsilon}, \widetilde{Z}_{r}^{\epsilon}\right)-U_{2}\left(r, r, Y_{r}^{\epsilon}, \nabla \bar{u}^{\epsilon}(r, \bar{r}) G(r, r)\right)\right] \mathrm{d} r \\
& +\int_{s}^{T}\left\{U_{1}\left(r, r, Y_{r}^{\epsilon}\right)+U_{2}\left[r, r, Y_{r}^{\epsilon}, \nabla \bar{u}^{\epsilon}(r, \bar{r}) G(r, r)\right]\right\} \mathrm{d} r-\int_{s}^{T} \widetilde{Z}_{r}^{\epsilon} \mathrm{d} B_{r} .
\end{aligned}
$$


On the other hand, let us write thanks to the Itô formula,

$$
\bar{u}^{\epsilon}(s, \bar{s})=g^{\epsilon}(\bar{T})+\int_{s}^{T}\left[-\partial_{r} \bar{u}^{\epsilon}(r, \bar{r})-L^{\infty, \epsilon} \bar{u}^{\epsilon}(r, \bar{r})\right] \mathrm{d} r-\int_{s}^{T} \nabla \bar{u}^{\epsilon}(r, \bar{r}) G(r, r) \mathrm{d} \widetilde{B}_{r},
$$

where we introduce the operator (see also eq. (1.3) and (3.2))

$$
L^{\infty, \epsilon}=\frac{1}{2} \sum_{i, j=1}^{d} \Lambda_{i j}\left(\frac{X_{r}^{\epsilon}}{\epsilon}, X_{r}^{\epsilon}\right) \partial_{x_{i} x_{j}}^{2}+\sum_{i=1}^{d} F_{i}\left(\frac{X_{r}^{\epsilon}}{\epsilon}, X_{r}^{\epsilon}, Y_{r}^{\epsilon}\right) \partial_{x_{i}} .
$$

Recall that the operator $\bar{L}(x,$.$) was defined in equations (3.5) and (3.7), so that we have in particular$

$$
\bar{L}\left(\bar{X}_{r}^{\epsilon}, .\right)=\frac{1}{2} \sum_{i, j=1}^{d} \bar{\Lambda}_{i j}\left(\bar{X}_{r}^{\epsilon}\right) \partial_{x_{i} x_{j}}^{2}+\sum_{i=1}^{d} \bar{F}_{i}\left(\bar{X}_{r}^{\epsilon}, \cdot\right) \partial_{x_{i}} .
$$

Hence we can write

$$
\begin{aligned}
\bar{u}^{\epsilon}(s, \bar{s})= & g^{\epsilon}(\bar{T})+\int_{s}^{T} \bar{\rho}_{1, r}^{\epsilon} \mathrm{d} r+\int_{s}^{T}\left[\bar{U}_{1}\left(\bar{r}, \bar{u}^{\epsilon}(r, \bar{r})\right)+\bar{U}_{2}\left(\bar{r}, \bar{u}^{\epsilon}(r, \bar{r}), \nabla \bar{u}^{\epsilon}(r, \bar{r})\right)\right. \\
& \left.-\left(L^{\infty, \epsilon}-\bar{L}(\bar{r}, .)\right) \bar{u}^{\epsilon}(r, \bar{r})\right] \mathrm{d} r-\int_{s}^{T} \nabla \bar{u}^{\epsilon}(r, \bar{r}) G(r, r) \mathrm{d} \widetilde{B}_{r}
\end{aligned}
$$

where

$$
\bar{\rho}_{1, r}^{\epsilon}=\left(\bar{U}_{2}^{\epsilon}-\bar{U}_{2}\right)\left(\bar{r}, \bar{u}^{\epsilon}(r, \bar{r}), \nabla \bar{u}^{\epsilon}(r, \bar{r})\right) .
$$

We have moreover (suppressing the trace symbol Tr)

$$
\begin{aligned}
\int_{s}^{T}\left(L^{\infty, \epsilon}-\bar{L}(\bar{r}, .)\right) \bar{u}^{\epsilon}(r, \bar{r}) \mathrm{d} r= & \frac{1}{2} \int_{s}^{T}[\Lambda(r, r)-\bar{\Lambda}(r)] \partial^{2} \bar{u}^{\epsilon}(r, \bar{r}) \mathrm{d} r+\int_{s}^{T}\left[F\left(r, r, Y_{r}^{\epsilon}\right)-\bar{F}\left(r, Y_{r}^{\epsilon}\right)\right] \nabla \bar{u}^{\epsilon}(r, \bar{r}) \mathrm{d} r \\
& +\int_{s}^{T}\left[\bar{F}\left(\bar{r}, \bar{Y}_{r}^{\epsilon}\right)-\bar{F}\left(\bar{r}, \bar{u}^{\epsilon}(r, \bar{r})\right)\right] \nabla \bar{u}^{\epsilon}(r, \bar{r}) \mathrm{d} r+\int_{s}^{T} \bar{\rho}_{2, r}^{\epsilon} \mathrm{d} r
\end{aligned}
$$

where

Now, with

$$
\bar{\rho}_{2, r}^{\epsilon}=\frac{1}{2}[\bar{\Lambda}(r)-\bar{\Lambda}(\bar{r})] \partial^{2} \bar{u}^{\epsilon}(r, \bar{r})+\left[\bar{F}\left(r, Y_{r}^{\epsilon}\right)-\bar{F}\left(\bar{r}, \bar{Y}_{r}^{\epsilon}\right] \nabla \bar{u}^{\epsilon}(r, \bar{r})\right.
$$

we can write

$$
\bar{\rho}_{r}^{\epsilon}=\bar{\rho}_{1, r}^{\epsilon}-\bar{\rho}_{2, r}^{\epsilon}
$$

$$
\begin{aligned}
\bar{u}^{\epsilon}(s, \bar{s})= & g^{\epsilon}(\bar{T})+\int_{s}^{T} \bar{\rho}_{r}^{\epsilon} \mathrm{d} r+\int_{s}^{T}\left[\bar{U}_{1}\left(r, Y_{r}^{\epsilon}\right)+\bar{U}_{2}\left(r, Y_{r}^{\epsilon}, \nabla \bar{u}^{\epsilon}(r, \bar{r})\right)\right] \mathrm{d} r \\
& -\int_{s}^{T}\left[\left(L^{\infty, \epsilon}-\frac{1}{2} \bar{\Lambda}(r) \partial^{2}(\cdot)-\bar{F}\left(r, Y_{r}^{\epsilon}\right) \nabla(\cdot)\right) \bar{u}^{\epsilon}(r, \bar{r})\right] \mathrm{d} r \\
& +\int_{s}^{T}\left[\bar{U}_{1}\left(\bar{r}, \bar{u}^{\epsilon}(r, \bar{r})\right)-\bar{U}_{1}\left(r, Y_{r}^{\epsilon}\right)\right] \mathrm{d} r \\
& +\int_{s}^{T}\left[\bar{U}_{2}\left(\bar{r}, \bar{u}^{\epsilon}(r, \bar{r}), \nabla \bar{u}^{\epsilon}(r, \bar{r})\right)-\bar{U}_{2}\left(r, Y_{r}^{\epsilon}, \nabla \bar{u}^{\epsilon}(r, \bar{r})\right)\right] \mathrm{d} r \\
& -\int_{s}^{T}\left[\bar{F}\left(\bar{r}, \bar{Y}_{r}^{\epsilon}\right)-\bar{F}\left(\bar{r}, \bar{u}^{\epsilon}\left(r, \bar{X}_{r}^{\epsilon}\right)\right)\right] \nabla \bar{u}^{\epsilon}(r, \bar{r}) \mathrm{d} r-\int_{s}^{T} \nabla \bar{u}^{\epsilon}(r, \bar{r}) G(r, r) \mathrm{d} \widetilde{B}_{r} .
\end{aligned}
$$


The natural idea is now to substract $\bar{u}^{\epsilon}(s, \bar{s})$ from $\bar{Y}_{s}^{\epsilon}$ rightaway and prove that this difference is small. However, we immediately run into technical difficulties which stem from the fact that the ergodic character of the second and third functionals on the right hand-side of the above relation would be destroyed by the use of rough inequalities. Another difficulty is that the second order derivatives of $\bar{u}^{\epsilon}(s, x)$ (in the space variables) explode as $\epsilon \rightarrow 0$. As far as the first problem is concerned, it is convenient to define first a few processes in order to shorten the above relations and substract $\bar{u}^{\epsilon}(s, \bar{s})$ from $\bar{Y}_{s}^{\epsilon}$ (modulo a crucial ergodic term, see (4.3) below). Define

where

$$
\delta_{r}^{\epsilon}=\delta_{1, r}^{\epsilon}+\delta_{2, r}^{\epsilon}+\delta_{3, r}^{\epsilon}
$$

$$
\begin{aligned}
& \delta_{1, r}^{\epsilon}=U_{1}\left(r, r, Y_{r}^{\epsilon}\right)-\bar{U}_{1}\left(r, Y_{r}^{\epsilon}\right), \\
& \delta_{2, r}^{\epsilon}=U_{2}\left(r, r, Y_{r}^{\epsilon}, \nabla \bar{u}^{\epsilon}(r, \bar{r}) G(r, r)\right)-\bar{U}_{2}\left(r, Y_{r}^{\epsilon}, \nabla \bar{u}^{\epsilon}(r, \bar{r})\right), \\
& \delta_{3, r}^{\epsilon}=\left[L^{\infty, \epsilon}-\frac{1}{2} \bar{\Lambda}(r) \partial^{2}(\cdot)-\bar{F}\left(r, Y_{r}^{\epsilon}\right) \nabla(\cdot)\right] \bar{u}^{\epsilon}(r, \bar{r}) .
\end{aligned}
$$

Define also

$$
\begin{aligned}
\delta Y_{s}^{\epsilon} & =\bar{Y}_{s}^{\epsilon}-\bar{u}^{\epsilon}(s, \bar{s})+\int_{t}^{s} \delta_{r}^{\epsilon} \mathrm{d} r \\
& =Y_{s}^{\epsilon}-\bar{u}^{\epsilon}(s, \bar{s})-\rho_{s}^{\epsilon}+\int_{t}^{s} \delta_{r}^{\epsilon} \mathrm{d} r \\
\delta Z_{s}^{\epsilon} & =\widetilde{Z}_{s}^{\epsilon}-\nabla \bar{u}^{\epsilon}(s, \bar{s}) G(s, s),
\end{aligned}
$$

see again (3.1) for the definition of the process $\rho^{\epsilon}$. We have therefore

$$
\begin{aligned}
\delta Y_{s}^{\epsilon}= & g(T)-g^{\epsilon}(\bar{T})+\int_{t}^{T} \delta_{r}^{\epsilon} \mathrm{d} r-\int_{s}^{T} \bar{\rho}_{r}^{\epsilon} \mathrm{d} r+\int_{s}^{T}\left[U_{2}\left(r, r, Y_{r}^{\epsilon}, \widetilde{Z}_{r}^{\epsilon}\right)-U_{2}\left(r, r, Y_{r}^{\epsilon}, \nabla \bar{u}^{\epsilon}(r, \bar{r}) G(r, r)\right)\right] \mathrm{d} r \\
& -\int_{s}^{T}\left[\bar{U}_{1}\left(\bar{r}, \bar{u}^{\epsilon}(r, \bar{r})\right)-\bar{U}_{1}\left(r, Y_{r}^{\epsilon}\right)\right] \mathrm{d} r-\int_{s}^{T}\left\{\bar{U}_{2}\left[\bar{r}, \bar{u}^{\epsilon}(r, \bar{r}), \nabla \bar{u}^{\epsilon}(r, \bar{r})\right]-\bar{U}_{2}\left[r, Y_{r}^{\epsilon}, \nabla \bar{u}^{\epsilon}(r, \bar{r})\right]\right\} \mathrm{d} r \\
& +\int_{s}^{T}\left[\bar{F}\left(\bar{r}, \bar{Y}_{r}^{\epsilon}\right)-\bar{F}\left(\bar{r}, \bar{u}^{\epsilon}(r, \bar{r})\right)\right] \nabla \bar{u}^{\epsilon}(r, \bar{r}) \mathrm{d} r-\int_{s}^{T} \delta Z_{r}^{\epsilon} \mathrm{d} \widetilde{B}_{r} .
\end{aligned}
$$

We now use the Itô formula, yielding

$$
\begin{aligned}
\left(\delta Y_{s}^{\epsilon}\right)^{2}+\int_{s}^{T}\left\|\delta Z_{r}^{\epsilon}\right\|^{2} \mathrm{~d} r= & \left\{\left[g(T)-g^{\epsilon}(\bar{T})\right]+\int_{t}^{T} \delta_{r}^{\epsilon} \mathrm{d} r\right\}^{2}-2 \int_{s}^{T} \bar{\rho}_{r}^{\epsilon} \delta Y_{r}^{\epsilon} \mathrm{d} r \\
& +2 \int_{s}^{T}\left[U_{2}\left(r, r, Y_{r}^{\epsilon}, \widetilde{Z}_{r}^{\epsilon}\right)-U_{2}\left(r, r, Y_{r}^{\epsilon}, \nabla \bar{u}^{\epsilon}(r, \bar{r}) G(r, r)\right)\right] \delta Y_{r}^{\epsilon} \mathrm{d} r \\
& -2 \int_{s}^{T}\left[\bar{U}_{1}\left(\bar{r}, \bar{u}^{\epsilon}(r, \bar{r})\right)-\bar{U}_{1}\left(r, Y_{r}^{\epsilon}\right)\right] \delta Y_{r}^{\epsilon} \mathrm{d} r \\
& -2 \int_{s}^{T}\left[\bar{U}_{2}\left(\bar{r}, \bar{u}^{\epsilon}(r, \bar{r}), \nabla \bar{u}^{\epsilon}(r, \bar{r})\right)-\bar{U}_{2}\left(r, Y_{r}^{\epsilon}, \nabla \bar{u}^{\epsilon}(r, \bar{r})\right)\right] \delta Y_{r}^{\epsilon} \mathrm{d} r \\
& +2 \int_{s}^{T}\left[\bar{F}\left(\bar{r}, \bar{Y}_{r}^{\epsilon}\right)-\bar{F}\left(\bar{r}, \bar{u}^{\epsilon}\left(r, \bar{X}_{r}^{\epsilon}\right)\right)\right] \nabla \bar{u}^{\epsilon}(r, \bar{r}) \delta Y_{r}^{\epsilon} \mathrm{d} r \\
& -2 \int_{s}^{T} \delta Y_{r}^{\epsilon} \delta Z_{r}^{\epsilon} \mathrm{d} \widetilde{B}_{r} .
\end{aligned}
$$


By (1.10), the first (Lebesgue) integrand on the right hand-side above is dominated (in absolute value) by

$$
\left|\bar{\rho}_{r}^{\epsilon}\right|^{2}+\left|\delta Y_{r}^{\epsilon}\right|^{2} .
$$

Since $U_{2}$ is Lipschitz with respect to the last variable, we see that the second integrand is majorized by

$$
c\left(\rho^{2}\left\|\delta Z_{r}^{\epsilon}\right\|^{2}+\rho^{-2}\left|\delta Y_{r}^{\epsilon}\right|^{2}\right),
$$

where $\rho$ is an arbitrary (small) positive number. From the relation (2.2) and the following inequality

$$
\left|\bar{u}^{\epsilon}(r, \bar{r})-Y_{r}^{\epsilon}\right| \leq\left|\int_{t}^{r} \delta_{u}^{\epsilon} \mathrm{d} u\right|+\left|\rho_{r}^{\epsilon}\right|+\left|\delta Y_{r}^{\epsilon}\right|,
$$

we can see that the three remaining integrands in the Lebesgue integrals are dominated by the quantity

$$
c\left(\left|\int_{t}^{r} \delta_{u}^{\epsilon} \mathrm{d} u\right|^{2}+\left\|R_{r}^{\epsilon}\right\|^{2}+\left|\rho_{r}^{\epsilon}\right|^{2}+\left|\delta Y_{r}^{\epsilon}\right|^{2}\right) .
$$

We thus deduce from the Gronwall-Bellman inequality

$$
\begin{gathered}
\widetilde{E} \sup _{t \leq s \leq T}\left|\delta Y_{s}^{\epsilon}\right|^{2}+\widetilde{E} \int_{t}^{T}\left\|\delta Z_{s}^{\epsilon}\right\|^{2} \mathrm{~d} s \leq \\
c\left[\widetilde{E}\left|g(T)-g^{\epsilon}(\bar{T})\right|^{2}+\widetilde{E}\left(\sup _{t \leq s \leq T}\left|\int_{t}^{s} \delta_{r}^{\epsilon} \mathrm{d} r\right|^{2}\right)\right. \\
\left.+\widetilde{E} \sup _{t \leq s \leq T}\left(\left|\bar{\rho}_{s}^{\epsilon}\right|^{2}+\left\|R_{s}^{\epsilon}\right\|^{2}+\left|\rho_{s}^{\epsilon}\right|^{2}\right)\right] .
\end{gathered}
$$

Thanks to Corollary 3.2, there remains only to show that the random variables

$$
\sup _{t \leq s \leq T}\left|\int_{t}^{s} \delta_{r}^{\epsilon} \mathrm{d} r\right| \quad \text { and } \sup _{t \leq s \leq T}\left|\bar{\rho}_{s}^{\epsilon}\right|
$$

tend to zero in $L^{2}(\widetilde{P})$.

Lemma 4.1. We have as $\epsilon \rightarrow 0$

$$
\widetilde{E} \sup _{t \leq s \leq T}\left|\bar{\rho}_{s}^{\epsilon}\right|^{2} \rightarrow 0 .
$$

Proof. Since the treatment of $\bar{\rho}_{1, s}^{\epsilon}$ is easy, we only deal with the term $\bar{\rho}_{2, s}^{\epsilon}$ (see (4.1)). By Lemma 1.3 it follows that both $\bar{\Lambda}$ and $\bar{F}$ are Lipschitz. By Lemma 3.1 and the relation (3.11) we can write,

$$
\widetilde{E} \sup _{t \leq s \leq T}\left|\bar{\rho}_{2, s}^{\epsilon}\right|^{2} \leq c \epsilon^{2}\left(c_{\nabla}^{2}+M^{2}(\epsilon)\right) .
$$

It remains only to treat the term above involving $\delta_{r}^{\epsilon}$. It suffices to apply the following ergodic theorem to deal with each of the components in $\delta_{r}^{\epsilon}$.

Theorem 4.2. We have as $\epsilon \rightarrow 0$

$$
\widetilde{E} \sup _{t \leq s \leq T}\left|\int_{t}^{s} \delta_{r}^{\epsilon} \mathrm{d} r\right|^{2} \rightarrow 0
$$

Recall the decomposition (4.2) and define

$$
\delta_{3, s}^{\epsilon}=\delta_{31, s}^{\epsilon}+\delta_{32, s}^{\epsilon}
$$


where

$$
\begin{aligned}
\delta_{31, s}^{\epsilon} & =\left[F\left(s, s, Y_{s}^{\epsilon}\right)-\bar{F}\left(s, Y_{s}^{\epsilon}\right)\right] \nabla \bar{u}^{\epsilon}(s, \bar{s}), \\
\delta_{32, s}^{\epsilon} & =\frac{1}{2} \operatorname{Tr}[\Lambda(s, s)-\bar{\Lambda}(s)] \partial_{x}^{2} \bar{u}^{\epsilon}(s, \bar{s}) .
\end{aligned}
$$

Let us postpone for a while the study of the term $\delta_{32, s}^{\epsilon}$, which is delicate since the derivatives $\partial_{x_{i} x_{j}}^{2} \bar{u}^{\epsilon}(s, x)$, $1 \leq i, j \leq d$, may explode at infinity, $(s, x) \in[0, T] \times R^{d}$. We first treat $\delta_{1, s}^{\epsilon}, \delta_{2, s}^{\epsilon}$ and $\delta_{31, s}^{\epsilon}$.

The convergence to zero in $\widetilde{P}$-Probability of

$$
\sup _{t \leq s \leq T}\left|\int_{t}^{s} \delta_{i, r}^{\epsilon} \mathrm{d} r\right|
$$

for $i=1,2,31$, follows from Lemma 4.2 of Pardoux [12]. Moreover, there exists a constant $c>0$ s.t. for all $\epsilon \leq 1$ we have a.s.

$$
\sup _{t \leq s \leq T}\left|\delta_{i, s}^{\epsilon}\right| \leq c
$$

for all $i=1,2,31$, by Lemma 1.3 and the boundedness of $a$, the process $Y^{\epsilon}$ (see (2.14)) and the gradient $\nabla \bar{u}^{\epsilon}$; whence the convergence to zero in $L^{2}(\widetilde{P})$.

On the other hand, to deal with the term $\delta_{32, s}^{\epsilon}$, we define the matrix

$$
h_{2}\left(x^{1}, x^{2}\right)=\Lambda\left(x^{1}, x^{2}\right)-\bar{\Lambda}\left(x^{2}\right),
$$

and set for $\left(x^{1}, x^{2}, s, x^{3}\right) \in[0, T] \times R^{3 d}$

$$
H_{2}^{\epsilon}\left(x^{1}, x^{2}, s, x^{3}\right)=\operatorname{Tr} h_{2}\left(x^{1}, x^{2}\right) \partial_{x^{3}}^{2} \bar{u}^{\epsilon}\left(s, x^{3}\right) .
$$

We have the

Lemma 4.3. The function

$$
H_{2}^{\epsilon}\left(x^{1}, x^{2}, s, x^{3}\right)
$$

defined for $\left(x^{1}, x^{2}, s, x^{3}\right) \in[0, T] \times R^{3 d}$, is periodic with respect to the first variable with period one in each direction of $R^{d}$ and satisfies (1.8). For each $(t, x)$ in $[0, T] \times R^{d}$

$$
\sup _{t \leq s \leq T}\left|\int_{t}^{s} H_{2}^{\epsilon}\left(\frac{X_{r}^{\epsilon}}{\epsilon}, X_{r}^{\epsilon}, r, \bar{X}_{r}^{\epsilon}\right) \mathrm{d} r\right| \rightarrow 0
$$

in $L^{2}(\widetilde{P})$ as $\epsilon \rightarrow 0$.

Proof. We want to apply the Itô formula. Since $h_{2}$ need not be smooth, let us make use of regularization. Consider the regularized function,

$$
h_{2}^{\epsilon}\left(x^{1}, x^{2}\right)=\eta(\epsilon)^{-2 d} \int_{R^{2 d}} h_{2}\left(x^{1}-x^{1 \prime}, x^{2}-x^{2 \prime}\right) \varphi\left(\frac{x^{1 \prime}}{\eta(\epsilon)}, \frac{x^{2 \prime}}{\eta(\epsilon)}\right) \mathrm{d} x^{1 \prime} \mathrm{d} x^{2 \prime},
$$

where $\varphi$ is a standard mollifier. We also define as in Section 2 of [3]

$$
\begin{aligned}
& a^{\epsilon}\left(x^{1}, x^{2}\right)=\epsilon^{-d} \int_{R^{d}} a\left(x^{1}, x^{2}-x^{2 \prime}\right) \varphi\left(\frac{x^{2 \prime}}{\epsilon}\right) \mathrm{d} x^{2 \prime}, \\
& b^{\epsilon}\left(x^{1}, x^{2}\right)=\epsilon^{-d} \int_{R^{d}} b\left(x^{1}, x^{2}-x^{2 \prime}\right) \varphi\left(\frac{x^{2 \prime}}{\epsilon}\right) \mathrm{d} x^{2 \prime} .
\end{aligned}
$$


The function $h_{2}^{\epsilon}$ need not be orthogonal to $\mu^{\epsilon}\left(\mathrm{d} x^{1}, x^{2}\right)$, the invariant probability measure for the diffusion $X_{s}^{\epsilon, x^{2}}$ on $T^{d}$ given by

with generator (see also (1.7))

$$
X_{s}^{\epsilon, x^{2}}=x^{1}+\int_{t}^{s} b^{\epsilon}\left(X_{r}^{\epsilon, x^{2}}, x^{2}\right) \mathrm{d} r+\int_{t}^{s} \sigma^{\epsilon}\left(X_{r}^{\epsilon, x^{2}}, x^{2}\right) \mathrm{d} \widetilde{B}_{r}
$$

$$
L_{x^{2}}^{\epsilon}=\frac{1}{2} \sum_{i, j=1}^{d} a_{i j}^{\epsilon}\left(x^{1}, x^{2}\right) \partial_{x_{i}^{1} x_{j}^{1}}^{2}+\sum_{i=1}^{d} b_{i}^{\epsilon}\left(x^{1}, x^{2}\right) \partial_{x_{i}^{1}} .
$$

That is why we solve the following Poisson problem

$$
L_{x^{2}}^{\epsilon} \widehat{h}_{2}^{\epsilon}\left(x^{1}, x^{2}\right)=\bar{h}_{2}^{\epsilon}\left(x^{2}\right)-h_{2}^{\epsilon}\left(x^{1}, x^{2}\right),
$$

where

$$
\bar{h}_{2}^{\epsilon}\left(x^{2}\right)=\int_{\mathbb{T}^{d}} h_{2}^{\epsilon}\left(x^{1}, x^{2}\right) p_{\infty}^{\epsilon}\left(x^{1}, x^{2}\right) \mathrm{d} x^{1},
$$

in which $p_{\infty}^{\epsilon}\left(x^{1}, x^{2}\right)$ stands for the density of $\mu^{\epsilon}\left(\mathrm{d} x^{1}, x^{2}\right)$. Next, we need to set

$$
h_{2, \epsilon}\left(x^{1}, x^{2}\right)=h_{2}\left(x^{1}, x^{2}\right)-h_{2}^{\epsilon}\left(x^{1}, x^{2}\right) .
$$

Note that

$$
\widehat{H}_{2}^{\epsilon}\left(x^{1}, x^{2}, s, x^{3}\right)=\widehat{h}_{2}^{\epsilon}\left(x^{1}, x^{2}\right) \partial_{x^{3}}^{2} \bar{u}^{\epsilon}\left(s, x^{3}\right),
$$

solves the above Poisson problem with the function $\left(\bar{h}_{2}^{\epsilon}-h_{2}^{\epsilon}\right) \partial_{x^{3}}^{2} \bar{u}^{\epsilon}$ as a source term.

Now, we define the discontinuous càdlàg semi-martingale $\left(s_{*(1)}, \bar{X}_{s_{*(1)}}^{\epsilon}\right)$ with values in $[0, T] \times R^{d}$. Thanks to the Itô formula for the above function $\widehat{H}_{2}^{\epsilon}\left(x^{1}, x^{2}, s, x^{3}\right)$ on the jump process $\left(\frac{X_{s}^{\epsilon}}{\epsilon}, X_{s}^{\epsilon}, s_{*(1)}, \bar{X}_{s_{*(1)}}^{\epsilon}\right)$ for $s \in[t, T]$, suppressing the Trace symbol Tr, the superscript 3 of $x^{3}$ and the $*(1)$ for convenience, we have

$$
\begin{aligned}
\int_{s}^{T} H_{2}^{\epsilon}\left(\frac{X_{r}^{\epsilon}}{\epsilon}, X_{r}^{\epsilon}, r, \bar{X}_{r}^{\epsilon}\right) \mathrm{d} r= & \int_{s}^{T} h_{2}(r, r) \Delta_{r_{*}, r} \partial_{x}^{2} \bar{u}^{\epsilon}\left(., \bar{X}_{.}^{\epsilon}\right) \mathrm{d} r+\int_{s}^{T}\left(L_{r}-L_{r}^{\epsilon}\right) \widehat{h}_{2}^{\epsilon}(r, r) \partial_{x}^{2} \bar{u}^{\epsilon}\left(r_{*}, \bar{X}_{r_{*}}^{\epsilon}\right) \mathrm{d} r \\
& +\int_{s}^{T} h_{2, \epsilon}(r, r) \partial_{x}^{2} \bar{u}^{\epsilon}\left(r_{*}, \bar{X}_{r_{*}}^{\epsilon}\right) \mathrm{d} r+\int_{s}^{T} \bar{h}_{2}^{\epsilon}(r, r) \partial_{x}^{2} \bar{u}^{\epsilon}\left(r_{*}, \bar{X}_{r_{*}}^{\epsilon}\right) \mathrm{d} r \\
& +\epsilon \int_{s}^{T}\left[\partial_{x^{1}} \widehat{h}_{2}^{\epsilon} b^{\prime}+\partial_{x^{2}} \widehat{h}_{2}^{\epsilon} b+\partial_{x^{1} x^{2}}^{2} \widehat{h}_{2}^{\epsilon} a\right](r, r) \partial_{x}^{2} \bar{u}^{\epsilon}\left(r_{*}, \bar{X}_{r_{*}}^{\epsilon}\right) \mathrm{d} r \\
& +\epsilon \int_{s}^{T} \partial_{x^{1}} \widehat{h}_{2}^{\epsilon} \sigma(r, r) \partial_{x}^{2} \bar{u}^{\epsilon}\left(r_{*}, \bar{X}_{r_{*}}^{\epsilon}\right) \mathrm{d} \widetilde{B}_{r} \\
& +\epsilon^{2} \int_{s}^{T}\left[\partial_{x^{2}} \widehat{h}_{2}^{\epsilon} b^{\prime}+\frac{1}{2} \partial_{x^{2}}^{2} \widehat{h}_{2}^{\epsilon} a\right](r, r) \partial_{x}^{2} \bar{u}^{\epsilon}\left(r_{*}, \bar{X}_{r_{*}}^{\epsilon}\right) \mathrm{d} r \\
& +\epsilon^{2} \int_{s}^{T} \partial_{x^{2}} \widehat{h}_{2}^{\epsilon} \sigma(r, r) \partial_{x}^{2} \bar{u}^{\epsilon}\left(r_{*}, \bar{X}_{r_{*}}^{\epsilon}\right) \mathrm{d} \widetilde{B}_{r} \\
& +\epsilon^{2} \sum_{t_{i}>s}^{T_{*}} \widehat{h}_{2}^{\epsilon}\left(t_{i}, t_{i}\right) \Delta_{t_{i-1}, t_{i}} \partial_{x}^{2} \bar{u}^{\epsilon}\left(., \bar{X}_{.}^{\epsilon}\right)+\epsilon^{2} \Delta_{T, s} \widehat{h}_{2}^{\epsilon}(., .) \partial_{x}^{2} \bar{u}^{\epsilon}\left(\cdot *, \bar{X}_{* *}^{\epsilon}\right) .
\end{aligned}
$$

We have by (3.10)

$$
\widetilde{E}\left\|\Delta_{r_{*}, r} \partial_{x}^{2} \bar{u}^{\epsilon}\left(., \bar{X}^{\epsilon}\right)\right\|^{2} \leq c N(\epsilon)^{2} \epsilon^{2 \beta}
$$


so that thanks to the relation (3.11) we have $N(\epsilon) \epsilon^{\beta} \rightarrow 0$ as $\epsilon \rightarrow 0$. Let us now consider the second term on the right-hand side above. Since $a, b$ and $h_{2}$ are Lipschitz, it follows that both the first and the second derivatives of $\widehat{h}_{2}^{\epsilon}$ with respect to $x^{1}$ are bounded. It then suffices to apply the relation (3.11). The treatment of the third term is also a consequence of the Lipschitz character of $h_{2}$. On the other hand, the computation in the proof of Lemma 7 in [3] and the relation (3.11) settle our fourth term. The fifth and the sixth terms are easy. As far as the seventh term is concerned, recall from Lemma 8 in [3] that we have

$$
\sup _{x^{2} \in R^{d}} \epsilon\left\|\partial_{x^{2}}^{2} p_{\infty}^{\epsilon}\left(., x^{2}\right)\right\|_{L^{2}\left(T^{d}\right)}<\infty
$$

so that by the Cauchy-Schwarz inequality, we can set an upper bound on the second order partial derivatives of $\bar{h}_{2}^{\epsilon}$; therefore provided $\eta(\epsilon)$ decreases to zero slowly enough, our seventh term tends to zero indeed by the relations (3.11) as $\epsilon \rightarrow 0$. The eighth term is easy. Next, since $\widehat{h}_{2}^{\epsilon}\left(x^{1}, x^{2}\right)$ is bounded, it remains only to notice that the jump term behaves like the first term since $\Delta t_{i}=\epsilon^{2}$; which proves our lemma.

Theorem 4.2 is now established.

From Corollary 3.2, Lemma 4.1 and Theorem 4.2 we have

$$
\sup _{t \leq s \leq T}\left|\delta Y_{s}^{\epsilon}\right| \rightarrow 0
$$

in $L^{2}(\widetilde{P})$ as $\epsilon \rightarrow 0$. Hence it clearly follows from equation (4.3) that

$$
\widetilde{E}\left|\bar{Y}_{t}^{\epsilon, t, x}-\bar{u}^{\epsilon}(t, x)\right| \rightarrow 0
$$

as $\epsilon \rightarrow 0$ from which Lemma 3.3 follows immediately.

Remark 4.4. It seems hard to work out estimates similar to those in the relation (3.11) when $g$ is in $\mathcal{C}^{0,1}(R)$, let alone $g$ continuous with polynomial growth at infinity. This technical problem, which stems from the fact that our limit PDE has become quite coupled and also depends explicitely on the linear growth of $x$ at infinity as a function of $u$ and $\nabla u$, prevents us from treating these cases as well.

Acknowledgements. The authors are thankful to Professor G. Barles for helpful discussions, to the r-eferee for his helpful comments and the first author expresses his gratitude for the hospitality and support of the LATP at University of Provence, Marseille, France and of CNRS under PICS 444.

\section{REFERENCES}

[1] G. Barles and E. Lesigne, SDE, BSDE and PDE. Pitman Res. Notes Math. 364 (1997) 47-80.

[2] A. Bencherif-Madani and E. Pardoux, Homogenization of a diffusion with locally periodic coefficients. Sém. Prob. XXXVIII, LNM 1857 (2003) 363-392.

[3] A. Bencherif-Madani and E. Pardoux, Locally periodic Homogenization. Asymp. Anal. 39 (2004) 263-279.

[4] A. Bensoussan, L. Boccardo and F. Murat, Homogenization of elliptic equations with principal part not in divergence form and Hamiltonian with quadratic growth. Comm. Pure. Appl. Math. 39 (1986) 769-805.

[5] R. Buckdahn, Y. Hu and S. Peng, Probabilistic approach to homogenization of viscosity solutions of parabolic PDEs. NoDEA Nonlinear Diff. Eq. Appl. 6 (1999) 395-411. 
[6] M.G. Crandall, H. Ishii and P.R. Lions, User's guide to viscosity solutions of second order partial differential equations. Bull. A.M.S. 27 (1992) 1-67.

[7] F. Delarue, On the existence and uniqueness of solutions to FBSDEs in a non-degenerate case. Stoch. Proc. Appl. 99 (2002) 209-286.

[8] F. Delarue, Auxiliary SDEs for homogenization of quasilinear PDEs with periodic coefficients. Ann. Prob. 32 (2004) $2305-2361$.

[9] A. Jakubowski, A non-Skorohod topology on the Skorohod space. Elec. J. Prob. 2 (1997) 1-21.

[10] T. Kurtz, Random time changes and convergence in distribution under the Meyer-Zheng conditions. Ann. Prob. 19 (1991) $1010-1034$

[11] P.A. Meyer and W.A. Zheng, Tightness criteria for laws of semimartingales. Anal. I. H. P. 20 (1984) 353-372.

[12] E. Pardoux, Homogenization of linear and semilinear second order Parabolic PDEs with periodic coefficients: -a probabilistic approach. J. Func. Anal. 167 (1999a) 498-520.

[13] E. Pardoux, BSDEs, weak convergence and homogenization of semilinear PDEs, in Nonlinear analysis, Differential Equations and Control, F.H. Clarke and R.J. Stern Eds., Kluwer Acad. Pub. (1999b) 503-549. 